
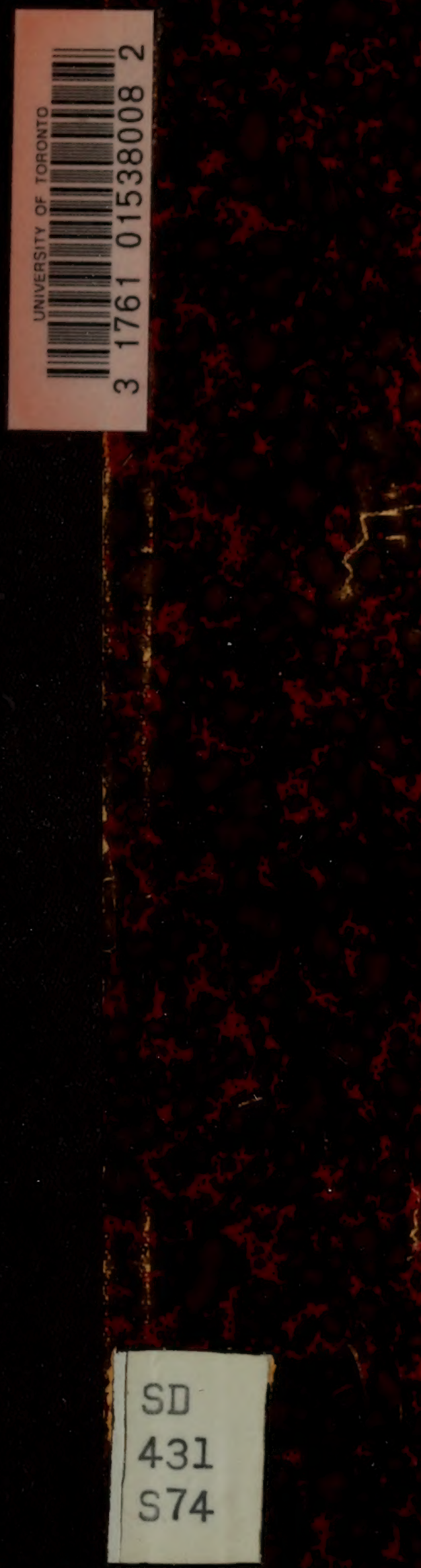

MWERSWY

$0 \%$

17Prid:

4WRARY 





\title{
Forsteinrichtung und
}

\section{Reservebildung}

\author{
mit besonderer Beziehung
}

auf die württembergischen Staatsforste.

Von

\section{Dr. Emil Speidel,}

Kgl. Forstrat in Stuttgart.

\section{LIBRARY}

FACULTY OF FORESTRY UNIVERSITY OF TORONTO
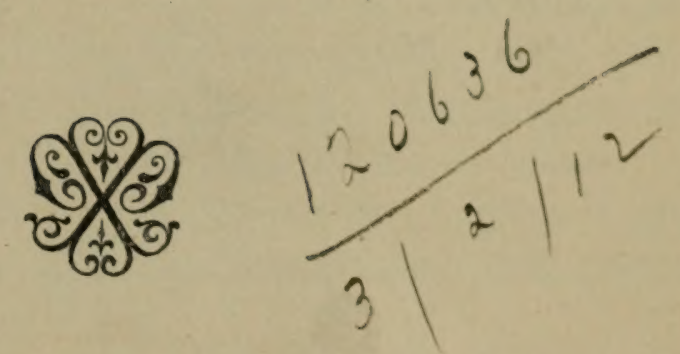

BERLIN.

Verlagsbuchhandlung Paul Parey.

Veriag für Landwirtsehaft, Gartenbau und Foratwesen.

SW., Hedemannstrasse 10.

1910. 
Alle Rechte, auch das der Übersetzung, vorbehalten.

$$
\begin{aligned}
& \text { SD } \\
& 431 \\
& 574
\end{aligned}
$$




\section{Vorwort.}

Zur Lösung zweier wichtiger Zeit- und Streitfragen: der Gestaltung des Forsteinrichtungswesens und der Fortbildung unseres Forstreservefonds - zu den Fragen, welche eben in Württemberg zu den brennenden gehören, - möchte ich in nachfolgendem Beiträge liefern. Hierbei werde ich Gelegenheit nehmen, auf den nahen inneren Zusammenhang, der zwischen den beiden Fragen besteht, hinzuweisen und den Weg zu zeigen, welcher bei richtiger Organisation des Einrichtungswesens mit der Zeit dazu führen dürfte, in unseren Nutzungen die strengere Scheidung zwischen Kapital und Zins mit hinreichender Genauigkeit zu erzielen und neben nachhaltigem Forstbetrieb auch befriedigende Finanzwirtschaft zu erreichen.

Die bezeichneten Fragen spielen übrigens zurzeit in ganz Deutschland eine Rolle! War es doch die Gestaltung des Forsteinrichtungswesens, welche bei der XVI. Tagung des Deutschen Forstwirtschaftsrats unter dem Titel: „Zeitgemäßes Forsteinrichtungsverfahren und die Einrichtung von besonderen Forsteinrichtungsämtern" beraten worden ist. Des weiteren glaube ich, daß der Vorgang Württembergs in der Reservebildung insbesondere dann, wenn eine Erweiterung des Reservefondsgesetzes in einer - im Interesse der Staatsforste selbst - verbesserten Auflage zustande gekommen sein wird, in sämtlichen Kulturstaaten mit größerem Waldbesitz Nachahmung finden wird.

Stuttgart, den 1. März 1910.

\section{Dr. Emil Speidel.}




\section{In halt.}

Seite

Einleitung . . . . . . . . . . . . . . . . . 5

Erstes Kapitel: Die periodische Nutzungsfläche und ihre Regelung 6 .

1. Die Flächenausgleichung nach rückwärts . . . . . . . 8

2. Die Flächenausgleichung nach vorwärts . . . . . . . . 10

Zweites Kapitel: Der periodische Massenvorrat und seine Abnutzung 15

1. Fall des Überalters der Abnutzungs-Bestände . . . . . . . 16

2. Fall des Unteralters der Abnutzungs-Bestände. . . . . . . . 19

Drittes Kapitel: Die Organisation des Forsteinrichtungswesens . 20

1. Die Ermittlung der Produktionsfaktoren (Inventaraufnahme) . . . 23

2. Die Feststellung vom Soll an Nutzung, Pflege und Kultur . . . 27

3. Die Organisation von Einrichtungsreferat und Einrichtungsbureau im besonderen . . . . . . . . . . . . . . . . 30

Viertes Kapitel: Die Reservebildung und ihre gesetzliche Regelung 33

1. Die Reserve und ihre Bestimmung im allgemeinen . . . . . . 33

2. Das bestehende württembergische Reservefondsgesetz . . . . . 36

3. Der weitere Ausbau der Fondsgesetzgebung. . . . . . . . 39

4. Die Füllung des Fonds und das Tempo hierfür . . . . . . . 40 


\section{Einleitung.}

Im Lauf von etwa mehr als 1 Jahrzehnt hat sich in der Forsteinrichtung der württembergischen Staats- und Körperschaftsforste der Übergang vom sogenannten kombinierten Fachwerk zur Flächenund Altersklassenmethode mit freier Bestandeswirtschaft, übrigens im Rahmen zweckmäßiger Hiebszugsbildung, vollzogen.

Der Übergang wurde sanktioniert durch die „Vorschriften für die Wirtschaftseinrichtung in den württembergischen Staats- und Körperschaftswaldungen" vom 6. Juli 1898.

Diese Vorschriften zeigen eine eigentümliche Zusammensetzung: einen Teil A: „Bisherige Anweisung“, in welchem eine 1878 als Entwurf ausgegebene „Anweisung betr. die Aufstellung, den Vollzug und die Erneuerung der Wirtschaftspläne für die Waldungen der Gemeinden, Stiftungen und sonstigen öffentlichen Körperschaften" wiedergegeben wird, weiterhin einen Teil B: „Neue Bestimmungen", durch welche der Teil A auf Grund der Erfahrung seit 1878 abgeändert und ergänzt wird.

Der Teil A gründet sich noch auf das kombinierte Fachwerk, der Teil B führt den Grundsatz der Altersklassenmethode ein, die Verschmelzung der verschiedenartigen Systeme dem geneigten Leser bezw. Forsteinrichter mehr oder weniger überlassend. Zeigen die Vorschriften so einen Januskopf, dessen eines Gesicht nach rückwärts zum alten System, nach der Abteilungswirtschaft blickt, das andere Gesicht nach vorwärts zum neuen System der Unterabteilungswirtschaft und der Zukunft entgegen, so haben sie auch noch für die Jetztzeit den Mangel, daß sie genauer Bestimmungen für Feststellung der Produktionsfaktoren, wie sie die Altersklassenmethode mit Bestandeswirtschaft erfordert, ferner der schärferen Regelung der periodischen Nutzungsfläche, der Vorratsabnutzung usw. entbehren.

Eine Erneuerung der Einrichtungsvorschriften ist daher als Bedürfnis zu bezeichnen, insbesondere im Interesse der Anfänger in der Kunst, welche in die Praxis der Forsteinrichtung eingeführt 
z. B. zur Feststellung der Produktionsfaktoren berufen werden. Es ist jedoch nicht meine Absicht, im nachfolgenden Vorschläge für die Erneuerung vom Einrichtungskodex in der systematischen Reihenfolge der Vorschriften von 1898 zu machen, diese verarbeitend, das Veraltete über Bord werfend und Neues zufügend, sondern ich werde den springenden Punkt der ganzen Einrichtungsaufgabe behandeln, das Einkommen aus den Wäldern zu bestimmen. Hiebei werde ich die Feststellung der dem nächsten Jahr-20 einer Betriebsklasse zukommenden Nutzungsfläche und die Abnutzung der auf ihr stockenden Holzvorräte nach forstlichen und finanziellen Gesichtspunkten in den Mittelgrund der Erörterungen stellen. Diese Feststellung tut zunächst not. Hierdurch gewinne ich zugleich die geeigneten Grundlagen für zweckmäßige Organisation des Einrichtungswesens einerseits, der Reservebildung andererseits.

\section{Erstes Kapitel.}

\section{Die periodische Nutzungsfläche und ihre Regelung.}

Die Einrichtungsvorschriften setzen in lit. B. „Neue Bestimmungen" Ziff. 7 und 8 folgendes fest:

,7) Zum Zweck der Ertragsregelung ist sodann unterabteilungsweise die Hiebsfläche auszuscheiden, welche die Grundlage des periodischen Nutzungsplans zu bilden hat.

In der Regel wird es genügen, die Hiebsfläche für die I. Periode speziell auszuscheiden.

8) Für das $\mathrm{Maß}$ der so auszuscheidenden Nutzungsflächen im ganzen bildet die Normalfläche einer 20 jährigen Periode den Hauptanhaltspunkt. Um jedoch den Anforderungen eines abnormen Altersklassenverhältnisses und eines abnormen Zustandes der Bestände genügend Rechnung tragen und abnorme Verhältnisse der einen oder andern Art auf kürzestem Wege und mit den geringsten Opfern verbessern zu können, sind ......Abweichungen von der Normalfläche zulässig, dergestalt, daß z. B. bei einem Abmangel an hiebsreifen Beständen die Nutzungsfläche der I. Periode unter den Betrag der Normalfläche herabgesetzt, dagegen bei einem Überschuß an hiebsreifen, desgleichen bei stärkerer Vertretung zuwachsarmer oder sonst unvollkommener Bestände über den Normalbetrag erhöht werden kann. - Derartige Abweichungen von dem normalen Soll sind uach Bedarf zu begründen."

Die Abweichungen von der Normalfläche eines Jahr-20 sind hiernach in das Ermessen des Einrichters gestellt und ein eigentliches $\mathrm{Maß}$ für dieselben ist nicht gegeben, insbesondere ist auch 
nicht rorgesehen, dal das Mals der Abnutzung in der Vergangen heit berücksichtigt wird.

Einen allgemeinen Maßstab) für jene Abweichungen zu schaffer, erscheint erwünscht, sowohl im Interesse der Einheitlichkeit der Bestimmungen, welche auf Herstellung einer normalen Altersstufenfolge abziclen, als auch mit Rücksicht auf die im II. Kapitol zu versuchende Anbahnung einer Scheidung der Ertrïge in solche det laufenden Verwaltung und solche eincr Reservererwaltung. In dieser Erwägung halte ich es auch für geboten, behufs Ausgleichung der Jahrzwauzigflächen nicht nur das Altersklassenverhältuis nach vorwärts d. h. in der Richtung ron den haubaren Bestïnden zu den angehend haubaren hin ins Auge zu fassen, sondern auch dasjenige nach rückwärts, nach den Jungbeständen hin.

Ein Mehrvorrat (Überschuß) an Jungbeständen gegenüber der Normalfläche stellt, abgesehen zunächst vom Zuwachs durch Erwerbung, eine Zurielnutzung der Vergangenheit dar, ein Wenigervorrat (Abmangel) an sólchen Beständen eine Mindernutzung dieser Vergangenheit.

Überschuß oder Abmangel der Jungbestïnde sind meines Erachtens bei Feststellung der periodischen Abnutzungsfläche ebenso. wenn unter Umständen auch nicht in gleichen Grad, zu berücksichtigen, wie Abweichungen der ältesten Altersklasse ron der Normalflüche und zwar dürften sie im Lauf eines rom Waldeigentümer zu bestimmenden Zeitraums auszugleichen sein, wenn auf strengere Nachhaltigkeit hingearbeitet werden soll, wie diese für die Staats- und Körperschaftswaldungen gefordert wird.

Um auf ein geeignetes Ma B der A usgleich ungen zu kommen, untersuche ich die rorkommenden Fälle im cinzelnen unter Benutzung ron Beispielen aus der Praxis. Hierbei wähle ich dic Bezeichnungen folgendermaßen:

für die Altersklassen von: 1-20 Jahren mit a, 21-40 mit b, 41-60 Jahren mit c, $61-80$ Jahren mit d, 81-100 Jahren mit $\theta$, über 100 Jahren mit $f$;

für die Bestockungsflächen dieser Altersklassen: die Ausdrüicke $\mathrm{F}_{\mathrm{a}}, \mathrm{F}_{\mathrm{b}}, \mathrm{F}_{\mathrm{c}}, \mathrm{F}_{\mathrm{l}}, \mathrm{F}_{\mathrm{o}}, \mathrm{F}_{\mathrm{f}}$, bezw. wenn nur 5 Altersklassen zu 20 Jahren ausgeschieden worden sind, für die älteste Klasse den Ausdruck $\mathrm{F}_{\mathrm{e}}$, endlich für die Normalflïche $\mathrm{F}_{\mathrm{n}}$ :

gebe ferner die Vertretung der betreffenden Altersklassen bezw. deren Flächenanteile in Prozenten der ertragsfähigen Fläche einer Betriebsklasse an. 


\section{Die Flächenausgleichung nach riickwärts}

(bezüglich der Jungflächen).

a) Fall des Überschusses an a-Beständen.

Haben wir das Altersklassenverhältnis, wie es sich z. B. im Forstbezirk Lorch 1903 bei 100 jähr. Umtrieb und für 5 Altersklassen ergeben hat:

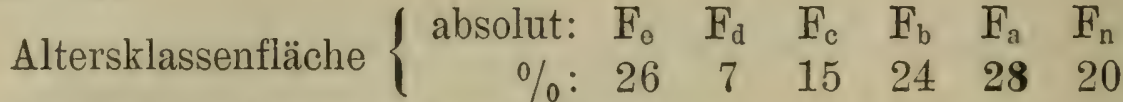

so liegt in dem Überschuß von $\mathrm{F}_{\mathrm{a}}$ gegenüber ron $\mathrm{F}_{\mathrm{n}}$ mit - : $8 \%$ - abgesehen von den örtlichen Gründen der Entstehung - eine Me hrnutzung im verausgegangenen Jahrzwanzig vor, welche bei Festsetzung der Nutzungsfläche des neuen Jahrzwanzig zu berücksichtigen ist.

Die Möglichkeit der Berücksichtigung ist eine verschiedene, je nachdem die Ausgleichung durch Verteilung des Überschusses auf die nächste Periode oder auf 2, 3 oder 4 Perioden, also auf den übrigen Teil der Umtriebszeit vorgenommen werden will. Wir erhielten bei Verteilung des Überschusses auf:

a) 1 Periode als Betreff einer Periode - : $8 \%$

ß) 2 Perioden, $, \quad, \quad \frac{8}{2}=4$,

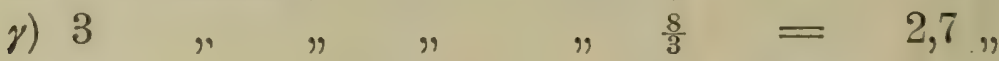

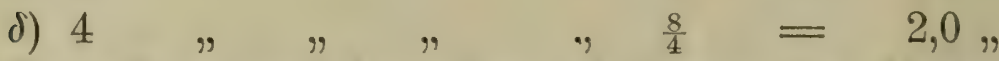

welcher Betreff zu berücksichtigen, d. h. an der Normalfläche des nächsten Jahrzwanzig (der I. Periode) abzuziehen wäre.

Es ergäbe sich als periodische Nutzungsfläche, welche ich mit $\mathrm{F}_{\mathbb{W}}$ bezeichnen will, im Fall:

$$
\begin{aligned}
& \text { ж) } \therefore 20-8,0 \%=12,0 \% \\
& \text { в) } \div 20-4,0, n=16,0,
\end{aligned}
$$

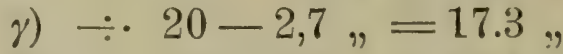

$$
\begin{aligned}
& \text { d) } \div 20-2,0, "=18,0,
\end{aligned}
$$

Bei Überschuß von a-Beständen wäre hiernach die normale Nutzungsfläche $\mathrm{F}_{\mathrm{n}}$ um einen von der Länge des gewählten Ausgleichungszeitraums bezw. der entsprechenden Zahl x ron Perioden abhängigen Betrag zu vermindern, oder, da die Differenz $F_{a}-F_{n}$ den Überschuß darstellt, allgemein ausgedrückt wäre:

$$
\mathrm{F}_{\mathrm{w}}=\mathrm{F}_{\mathrm{n}}-\frac{\mathrm{F}_{\mathrm{a}}-\mathrm{F}_{\mathrm{n}}}{\mathrm{x}}
$$

b) Fall des Abmangels an a-Beständen:

Haben wir hier das Aitersklassenverhältnis, wie es z. B. für das Forstamt Heimerdingen 1907 festgestellt worden ist: 
Altersklassenfläche $\left\{\begin{array}{ccccccc}\text { absolut: } & F_{\theta}+ & F_{d} & F_{c} & F_{b} & F_{a} & F_{n} \\ \text { in } \%: & 20 & 22 & 22 & 23 & 13 & 20\end{array}\right.$

so zeigt sich ein Abmangel in Altersklasse a bezw. eine Mindernutzung des letzten Jahrzwanzig von $7 \%$ dor Gesamtfläche.

Dieser Abmangel dürfte, obgleich die $F_{e}$-Flïche normal ist, bei Feststellung der Nutzungsfläche des neuen Jahrzwanzig ( $\mathrm{F}_{\mathrm{w}}$ ) zu berücksichtigen sein und kann analog wie der Überschul in verschiedener Weise ausgeglichen werden, je nachdem ich den Zeitraum für die Ausgleichung bestimme bezw. in 1, 2, 3 oder 4 Perioden durch entsprechende Mehrnutzung an Fläche ausgleiche.

Bei Verteilung des Abmangels auf je 1, 2, 3 oder 4 Perioden ergeben sich nachstehende Abnutzungsflächen:

a) bei 1 Periode $=7,0 \%$ pro Periode, Fläche $=20+7,0=27,0 \%$ $\left.\beta^{2}\right), 2, \quad=\frac{7}{2}=3,5, ", \quad, \quad, \quad=20+3,5=23,5$,

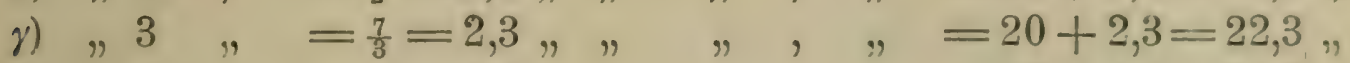
d) $" 4 " \quad=\frac{7}{4}=1,7 ", \quad, \quad, \quad=20+1,7=21,7$,

Hiernach ist die Normalfläche $\mathrm{F}_{\mathrm{n}}$ um einen Flächenbetrag $\mathrm{zu}$ erhöhen, welcher von der Zahl $x$ der für Ausgleichung gewählten Perioden oder Jahrzwanzig abhängt und sich, da der Abmangel in der Differenz $\mathrm{F}_{\mathrm{n}}-\mathrm{F}_{\mathrm{a}}$ gegeben ist, ausspricht in der Formel:

$$
\mathrm{F}_{\mathrm{w}}=\mathrm{F}_{\mathrm{n}}+\frac{\mathrm{F}_{\mathrm{n}}-\mathrm{F}_{\mathrm{a}}}{\mathrm{x}}
$$

(Formel II)

\section{c) Folgerungen für Ziffer 1 .}

Aus den Betrachtungen in lit. a und b läßt sich für die Ausgleichungen der Satz folgern:

Um Abweichungen der Altersklasse a rom Normal-Flächensoll auszugleichen, ist im Fall des Überschusses ein Abzug an der Normal-Fläche des nächsten Jahrzwanzig, im Fall des Abmangels ein Zuschlag zu letzterer Fläche zu machen.

Die Größe von Abzug wie von Zuschlag hängt ron der Frage ab, auf wieviele Jahrzwanzig die Differenz zwischen Soll und Hat verteilt werden will, mit andern Worten daron, ob die normale Altersstufenfolge in kürzerer oder längerer Zeit hergestellt werden will.

Der allgemeine Ausdruck für Bestimmung der Abnutzungsfläche des nächsten Jahrzwanzig mit Rücksicht auf die Vertretung der a-Altersstufe lautet, da: wenu $\mathrm{F}_{\mathrm{a}}>\mathrm{F}_{\mathrm{n}}$

$$
\mathrm{F}_{\mathrm{w}}=\mathrm{F}_{\mathrm{n}}-\frac{\mathrm{F}_{\mathrm{a}}-\mathrm{F}_{\mathrm{n}}}{\mathrm{x}} \text {, und }
$$

wenn $\mathrm{F}_{\mathrm{a}}<\mathrm{F}_{\mathrm{n}}$ 


$$
\begin{gathered}
\mathrm{F}_{\mathrm{w}}=\mathrm{F}_{\mathrm{n}}+\frac{\mathrm{F}_{\mathrm{n}}-\mathrm{F}_{\mathrm{a}}}{\mathrm{x}}: \\
\mathbf{F}_{\mathbf{w}}=\mathbf{F}_{\mathbf{n}}-\frac{\mathbf{F}_{\mathrm{a}}-\mathbf{F}_{\mathrm{n}}}{\mathbf{x}}
\end{gathered}
$$

Unter Anwendung dieser Formel ergäbe sich für: Lorch (Beisp. lit. a), 100 jähr. Umtrieb und $x=4:^{1}$ )

$$
\mathrm{F}_{\mathrm{w}}=20-\frac{28-20}{4}=18 \%
$$

Heimerdingen (Beisp. lit. b) 100 jähr. Umtrieb und $x=4$ :

$$
\mathrm{F}_{\mathrm{W}}=20-\frac{13-20}{4}=21,7 \% \text {. }
$$

\section{Die Flächenausgleichung nach rorwårts}

(bezüglich der Altflächen).

Auch hier ist zunächst wieder zu unterscheiden, ob in der ältesten Altersklasse, als welche

bei 120 jähr. Umtrieb die Fläche der $\mathrm{f}+$ Bestände,

$$
\begin{aligned}
& \text { " } 100 " \quad " \quad " \quad \text { " } \quad " \quad \text { + } \\
& " 80, \quad " \quad, \quad " \quad, d+
\end{aligned}
$$

angesehen" wird, ein Flächen - Überschuß oder -Abmangel rorhanden ist.

a) Fall des Überschusises von Altbeständen.

Die Größe dieses Überschusses ist unter Zugrundelegung der nach Ziff. 1c berechneten Abnutzungsfläche, also der nach dem Flächenanteil der a-Bestände regulierten Normalfläche, festzustellen.

Hat sich im Beispiel vom Forstamt Lorch ergeben: $F_{\mathrm{w}}=18 \%$ und ist ein Flächenbestand an Altholz (Alter e und mehr) vorhanden von: $\mathbf{F}_{\mathrm{e}}=\mathbf{2 6} \%$, so ergibt sich ein ÜberschuB der Altersklassenfläche e mit $\therefore 8 \%$ oder $\mathrm{F}_{\mathrm{e}}-\mathrm{F}_{\mathrm{W}}=+8 \%$.

Es erhebt sich die Frage, ob dieser Altholzüherschuß (an Fläche) einfach der nächst jüngeren Altersstufe (hier den d-Beständen) zur Nutzung überlassen wird und somit bis zum Beginn der letzteren

1) In der Regel wird zu setzen sein:

$$
\begin{aligned}
& \mathrm{x}=5 \text { bei } 120 \text { jährigem Umtrieb. } \\
& \mathrm{x}=4,5 \quad, 110 \quad, \quad \text { " } \\
& x=4 \quad " 100 \quad " \quad \text { " } \\
& \mathrm{x}=3,5 \quad, \quad 90 \quad, \quad, \\
& \mathrm{x}=3 \quad, \quad 80 \quad, \quad,
\end{aligned}
$$


stehen bleiben, un weitere 20 Jahre älter werden darf odex ob gänzliche oder endlich, ob teilweise Abnützung im nächsten Jahrzwanzig vorzuziehen ist.

Die Entscheidung über diese Frage hängt von Gründen teils forstlicher, teils finanzieller Natur ab, welche im Einzelfalle zu untersuchen und nach ihrem Gewicht abzuwägen sind.

In forstlicher Hinsicht kommt in Betracht: der Grarl der Hiebsreife der Bestände bezw. der mutmaßliche Massen- und Sortimentsanfall, die Rücksicht auf die natürliche Verjüngung und den augenblicklichen Stand derselben, auf die Hiebsfolge bezw. die Zugehörigkeit zu bestimmten Hiebszügen u. a.

In fin anzieller Hinsicht fragt es sich, ob ferneres Üborhalteu der überschüssigen Altbestände um 20-30 Jahre zur etwaigen Deckung eines Abmangels der d-Altersstufenfläche rom Standpuntt der Rentabilitätslehre, der genügenden Verzinsung des Produktionsaufwandes aus empfohlen werden kann oder nicht?

Im einzelnen sind folgende Möglichkeiten zu erörtern:

a) Zurückschiebung der Altbestände zulässig: Haben die forstlich-finanziellen Erwägungen ergeben, daß eine Verschiebung der überschüssigen Altbestände in das 2. Jahrzwanzig zulässig erscheint, so bildet die nach Formel III berechnete Abnutzungsfläche das Nutzungssoll des nächsten Jahrzwanzig, der Überschuß der Altbestände geht ohne weiteres dem 2. Jahrzwanzig zur ordnungsmäßigen Ausstattung der Soll-Fläche desselben zu.

Bei der Verschiebung der einzelnen Bestände wird darauf auszugehen sein, die jüngsten der Altbestände hierfür auszusuchen, wofür auch waldbauliche fründe meistens sprechen werden.

Im vorliegenden Fall wird der Verbesserung der Altersstufenfolge am besten Rechnung getragen, allerdings zugleich eine Art Materialreserve in überalten Beständen für die Abnutzungsflüche des 2. Jahrzwanzig geschaffen. Wie die Gelderträge dieser Bestände später in finanzieller Hinsicht zu behandelu sein werden, ist aus dem Kapitel II zu entnehmen.

Die erörterte Möglichkeit dürfte in der Hauptsache in Betriebsklassen mit niedrigen Umtrieben, etwa von 80 und 90 Jahren rorhanden sein.

ß) Zurückschiebung der Altbestände unzulässig: Weisen sämtliche Erwägungen darauf hin, daß eine Hinausschiebung der überschüssigen Altbestände sich nicht empfiehlt, so sind dieselben zur Abnutzung im nächsten Jahrzwanzig rorzusehen. Dieser Nutzung kommt jedoch ein anderer Charakter zu als der nach 
Ziff. 1 berechneten, der Charakter der Vorausnutzung von einer Fläche, deren Abnutzung ordnungsmäßig erst dem 2. Jahrzwanzig zustehen würde, d. h. der Charakter der auBerordentlichen Nutzung mit der Bestimmung des Geldertrags, einen etwaigen Ausfall an Nutzungsfläche bezw. Geld des 2. Jahrzwanzig zu decken. Es liegt hier eine Grundstocksabnutzung vor, deren Ertrag wieder einem Grundstock, einem Reservefonds, zuzuführen ist.

Bei derartiger áußerordentlicher Nutzung wird auf raschere Herstellung normaler Altersstufenfolge vorerst verzichtet, um nicht zu große Opfer, insbesondere finanzieller Natur bringen zu müssen.

Der unter $\beta$ besprochene Fall liegt bei der Mehrzahl der Altbestand-Überschüsse des Schwarzwalds vor, ferner aber auch in dem Beispiel rom Forstamt Lorch, wo sich die Abnutzung des Überschusses von $8 \%$ in außerordentlicher Weise entweder ganz oder teilweise empfiehlt, jedoch erst dann, wenn die Möglichkeit der Anlage des Geldertrags in einem Reservefonds gegeben ist.

$\gamma)$ Zurückschiebung teils zulässig, teils unzulässig: Ergeben die forstlich-finanziellen Erwägungen, daß für einen Teil der überschüssigen Altbestände die Zurückschiebung zulässig erscheint, für einen Teil nicht, so ist im ersteren Falle nach lit. $\alpha$, im letzteren nach lit. $\beta$ zu verfahren, bezw. das Soll der Nutzungsfläche des nächsten Jahrzwanzig entweder nach Formel III zur ordentlichen Nutzung zu bestimmen oder neben dieser noch eine Zusatzfläche zur außerordentlichen Nutzung vorzusehen.

Dieser Fall wird in der Praxis die Regel bilden und es ist jeweils darüber zu verfügen, welcher Teil der überschüssigen Altbestände, im Lorcher Beispiel der $8 \%$, zu nutzen, welcher zu belassen ist, welcher Betrag der Gesamtnutzung als ordentlicher, welcher als außerordentlicher Ertrag anzusehen ist.

b) Fall des Abmangels von Altbeständen.

Die Größe des Abmangels ist wie diejenige des Überschusses nach Maßgabe der nach dem Flächenanteil der a-Bestände regulierten Normalfläche zu berechnen.

Haben wir das Altersklassenverhältnis, wie es z. B. das Forstamt Gaildorf für 100 jähr. Umtrieb aufweist:

Flächenanteil der Altersklasse:

$\mathrm{e}+$

d

C

b

in Prozenten der ertragsfähigen Fläche
19

20

26 a 
so erhalten wir nach Formel III und wenn $\mathrm{x}=4$ :

$$
\mathrm{F}_{\mathrm{w}}=20-\frac{21-20}{4}=19,75 \%
$$

und bei $\mathrm{F}_{0}=$. . . . . . . - $14,0 \%$

einen Abmangel von $\theta$ mit . . - - 5,7,

oder:

$$
\mathrm{F}_{\Theta}-\mathrm{F}_{\mathrm{W}}=-5,7 \% \text {. }
$$

Hier fragt es sich nun, ob und inwieweit dieser $A$ bmangel aus jüngeren Beständen, denjenigen der d-Altersklasso gedeckt werden kann, ohne forstliche und finanzielle Rücksichten zu schädigen. An sich wäre solche Deckung im Interesse der baldigen Herbeiführung eines normalen Altersklassenverhältnisses wünschenswert.

Bei Beurteilung dieser Frage spieien wiederum forstliche und finanzielle Erwägungen herein: forstlicherseits sind Holzart, Standort, Betriebsweise, Altersklassenverhältnis usw. von Bedeutung.

Bezüglich der Holzart bedingt es einen Unterschied, ob das Nadelholz überwiegt oder das Laubholz. In der Regel wird nur bei Nadelholz und zwar bei Fichte und Tanne, jedoch bei Fichte eher, insbesondere auch bei Mischbeständen der beiden Holzarten mit Vorwiegen der Fichte, Deckung aus d-Beständen d. h. Vorschiebung solcher zulässig erscheinen.

Der Standort kommt insofern in Betracht, als bei geringerer Bonitüt die Möglichkeit der Vorschiebung weniger vorliegt als bei guten Standorten, wo in kürzerer Zeit brauchbare Sortimente heranwachsen, die natürliche Verjüngung leichter ronstatten geht usw.

Die seitherige Betriebsweise und Behandlung spielt auch eine Rolle, da es sich fragt, ob die Bestände seither regelrecht durchforstet worden sind, der Zuwachs so gefördert ist, daß marktgüngige Stammholzklassen anfallen, daß weiterhin die Bestände für natürliche Terjüngung richtig vorbereitet sind.

Wäre die Hauptfrage nach den rorgenaunten Gesichtspunkten zu bejahen, so ist auch noch das weitere Altersklassenverhältnis der Betriebsklasse ins Auge zu fassen, besonders die Vertretung der c-Altersklasse. Wenn die letztere wesentlichen Überschuß aufweist, so kann es sich fragen, ob nicht zur Deckung des Abmangels der Altbestände stärker in die d-Bestände eingegriffen werden sollte, als es sonst der Fall wäre.

In finanzieller Hinsicht ist zu bericksichtigen, daß in den 
d-Beständen relativ zu junges Holz, dessen Einheitswert unter demjenigen der Altbestände steht, genutzt wird. Bei Fichte und Tanne dürfte dieses Bedenken vom Standpunkt der Rentabilitätslehre aus zurücktreten, bei Forche und bei den Laubhölzern, zumal auch Nutzhölzern, zu würdigen sein.

Der finanzielle Abmangel, welchen die vorzuschiebenden dBestände event. mitbringen, wird vielfach dadurch ausgeglichen werden, daß die Altbestände ein höheres Alter haben, als die Umtriebszeit bedingen würde und damit auch größeren Holzvorrat, auf welchen Punkt ich in Kapitel II des näheren eingehen werde.

Schließlich noch die Bemerkung, daß im Fall der Vorschiebung von d-Beständen womöglich nur solche gewählt werden, welche an der oberen Grenze des d-Alters stehen.

\section{c) Folgerungen für Ziffer 2.}

Um Flächenabweichungen der Altersklasse e $\left(\mathrm{F}_{\mathrm{e}}\right)$ bezw. $\mathrm{f}\left(\mathrm{F}_{\mathrm{f}}\right)$ von dem nach dem Stand der a-Fläche regulierten Soll $\left(\mathrm{F}_{\mathrm{w}}\right)$ auszugleichen, ist im Fall des Überschusses von Altbeständen ein Zuschlag zu der berechneten $\mathrm{F}_{\mathrm{w}}$-Fläche begründet, im Fall des Abmangels solcher Bestände ein Abzug an der bezeichneten Fläche.

Die Größe des Zuschlags bezw. Abzugs hängt von waldbaulichfinanziellen Erwägungen über Möglichkeit und Zweckmäßigkeit von Verschiebungen je nach Holzart, Standort, Bestandesbeschaffenheit usw. ab.

Das Maß der hieraus sich ergebenden Verschiebungen kann in einem Bruchteil der Differenz:

$$
\mathrm{F}_{\theta}-\mathrm{F}_{\mathrm{w}}
$$

ausgedrückt werden, welche im Fall des Zuschlags positiv, im Fall des Abzugs negativ ist. Wird jener Bruchteil der Flächendifferenz mit y bezeichnet, so erhalten wir als endgültig regulierte Fläche $\left(\mathrm{F}_{\mathrm{w} 1}\right)$ :

wenn $\mathrm{F}_{\theta}>\mathrm{F}_{\mathrm{W}}$

wenn $\mathrm{F}_{0}<\mathrm{F}_{\mathrm{w}}$

$$
\mathrm{F}_{\mathrm{w} 1}=\mathrm{F}_{\mathrm{w}}+\frac{\mathrm{F}_{\mathrm{o}}-\mathrm{F}_{\mathrm{w}}}{\mathrm{y}}
$$

oder allgemein:

$$
\mathrm{F}_{\mathrm{w} 1}=\mathrm{F}_{\mathrm{w}}-\frac{\mathrm{F}_{\mathrm{w}}-\mathrm{F}_{\mathrm{e}}}{\mathrm{J}}
$$

$$
\mathbf{F}_{\boldsymbol{w} 1}=\mathbf{F}_{\boldsymbol{w}}+\frac{\mathbf{F}_{\mathrm{e}}-\mathbf{F}_{\mathbf{w}}}{\mathbf{y}}
$$


Das Bestreben wird vielfach dahin gehen, die Differenz: $\mathrm{F}_{6}-\mathrm{F}_{*}$ gleich zwischen den nächsten 2 Jahrzwanzig zu verteilen bezw, $y=2$ zu setzen. Auf dieser Grundlage würde sich die Nutzungsfläche fürs nächste Jahrzwanzig berechnen:

in dem Beispiel von Lorch und bei $\mathrm{x}=4$ :

$$
\mathrm{F}_{\mathrm{w}_{1}}=20-\frac{28-20}{4}-\frac{18-26}{2}=18+4=22 \%
$$

in dem Beispiel von Gaildorf und bei $\mathrm{x}=4$ :

$$
\mathrm{F}_{\mathbb{w}_{1}}=20-\frac{21-20}{4}-\frac{19,7-14,0}{2}=19,75-2,85=16,9 \%
$$

in wolch letzterem Fall - : 14,0\% aus den vorhandenen e-Beständen, der Rest mit 2,9\% auf Vorschiebung von d-Beständen kommen würde. ${ }^{1}$ )

Im Fall des Flächenüberschusses der Altbestände ergibt sich aus der Flächenformel $\mathbf{F}_{w_{1}}$ auch die Möglichkeit der Trennung der Bestände nach ihrem finanziellen Charakter $d$. h. der Bestände, deren Erträge als ordentliche Nutzung der laufenden Verwaltung zukommen, und der Bestände, deren Erträge eine Vorausnutzung für später, eine außerordentliche Nutzung darstellen und einer Reserveverwaltung zuzuweisen sind bezw. der Größe $\mathrm{F}_{\mathrm{w}}$ einerseits, der Größe $\frac{F_{\theta}-F_{W}}{y}$ andererseits.

\section{Zweites Kapitel.}

\section{Der periodische Massenvorrat und seine Abnutzung.}

In den Ausführungen von Kapitel I sind nicht nur die Grundlagen für Regelung der periodischen Abnutzungsfläche ron Fall zu Fall gegeben und damit für Anbahnung eines geregelten Alters-

1) Für die verschiedenen Möglichkeiten der Verteilung der Flächendifferenz $F_{0}-F_{W} z$ wischen dem I. und Il. Jahrzwanzig läßt sich nachstehende Tabelle der $\mathrm{y}$-Worte aufstellen:

\begin{tabular}{|c|c|c|c|c|c|}
\hline$"$ & $"$ & $"$ & $1: 3$ & " & $\mathrm{y}=4,0$ \\
\hline 9 & " & $"$ & $1: 2$ & $"$ & $\mathrm{y}=3,0$ \\
\hline 9 & $"$ & " & $1: 1$ & $"$ & $\bar{y}=2,0$ \\
\hline & $"$ & $"$ & $2: 1$ & " & $\mathrm{y}=1,5$ \\
\hline & $"$ & $"$ & $3: 1$ & $"$ & $\mathrm{y}=1,3$ \\
\hline & $"$ & , & $4: 1$ & $"$ & $\mathrm{y}=1,25$ \\
\hline
\end{tabular}

bei Verteilung wie $1: 4$ ist $\mathrm{y}=5,0$ 
klassenverhältnisses, sondern auch diejenigen für Ausscheidung zwischen ordentlicher und außerordentlicher Nutzung bezw. normaler Grundstocknutzung und Grundstocksabtragung, was insbesondere auch im Hinblick auf den Zusammenhang mit Bildung von Geldreserven des näheren untersucht werden will.

Im sogenannten Normalwald soll die Jahresnutzung dem jährlichen Gesamtzuwachs der ertragsfähigen Fläche und bei normalem Zuwachs dem Holzgehalt der ältesten Alterstufe vom normalen Alter der Umtriebszeit (u) entsprechen, also die Jahrzwanzig-Nutzung, für welche wir aus praktischen Gründen unsere Ertragsberechnungen anstellen, dem Holzgehalt bezw. normalen Vorrat der ältesten 20 jähr. Altersklasse bezw. von deren Fläche.

Nun haben aber in den wenigsten Fällen die der Nutzungsfläche des nächsten Jahrzwanzig nach Kapitel I zugeschiedenen Bestände das Normalalter der Umtriebszeit. Die eingestellten Bestände haben teils ein höheres Alter und damit in der Regel auch größere Massenvorräte, als der Umtriebszeit zukommen würden und für welche die Frage des Vorratsüberschusses zu erörtern ist, teils sind sie jünger, mußten zur Ergänzung aus der nächst jüngeren Altersklasse herangezogen werden. Im ersteren Fall haben wir Bestände mit Überalter z. B. in Lorch (Kapitel I), im letzteren Fall solche mit Unteralter, wie in Gaildorf. Die Wirkung dieser abnormen Altersverhältnisse, welchə jedoch in Wirklichkeit die Regel bilden, ist zugleich mit Rücksicht auf Ausscheidung von Vorratsüberschüssen und auf die finanzielle Behandlung vom Geldertrag der letzteren zu erörtern.

\section{Fall des Überalters der Abnutzungs-Bestïnde}

(Alter $\mathrm{a}=\mathrm{u}+\mathrm{z}$ ).

Bezeichnen wir die Anzahl der Jahre, um welche das tatsächliche Alter (a) dasjenige der Umtriebszeit (u) übersteigt, mit z, so ergibt sich folgendes:

a) Bei Flächenüberschuß der Althölzer

$$
\text { (wenn } \mathrm{F}_{\theta+}>\mathrm{F}_{\mathrm{n}} \text { ). }
$$

Von der nach Kapitel I als ordentlicher Nutzung berechneten Flächengröße ist nur derjenige Teil des Massenrorrats, welcher der normalen Umtriebszeit u entspricht, als ordentliche Vorratsnutzung zu betrachten, der übrige Teil ist außerordentlicher Massenvorrat, Ersparnis von früher, Vorratsüberschuß im eigent- 
lichen Sinn, welcher der laufenden Verwaltung streng genommen nicht zukommt, als außerordentliche Nutzung ausgeschieden werden kann.

Bei der zur a u ßerordentlichen Nutzung kommenden Flächengröße ist hinsichtlich des Massenvorrats zu unterscheiden:

a) der Massenanteil, welcher dem Alter d der nächst jüngeren Altersklasse bezw. dem Alter u -20 entspricht und dessen Fläche eigentlich dem Jahrzwanzig der jetzigen d-Bestände zukommen würde, und

B) der Massenanteil, welcher dem Alter a-d bezw. $a-(u-20)=a-u+20=z+20$ entspricht d. h. dem Zuwachs von $\mathrm{z}+20$ Jahren.

Der Ertrag des ersteren Massenanteils ist kein Vorratsüberschul, sondern hätte später im 2. Jahrzwanzig den Ertrag aus dem Vorrat der jetzigen d-Bestände im Alter u voraussichtlich zu ergänzen, wenn sich ein Abmangel an solchen Beständen künftig ergeben wird. Der Ertrag dieses Massenanteils hat zur Deckung späteren Ausfalls zu dienen und dürfte mit Rücksicht hierauf verzinslich anzulegen sein. Der Rest dagegen kann als Vorratsüberschuß angesehen und wie derjenige der ordentlichen Flächenabnutzung behandelt werden.

Um die Trennung zwischen ordentlicher und außerordentlicher Flächenabnutzung; zwischen Nutzung von ordentlichem und außerordentlichem Massenanteil, ferner die Zuscheidung der Gelderträge der betreffenden Flächenabnutzungen und Massenanteile zur laufenden Verwaltung einerseits, zur Reserve andererseits zu verdeutlichen, stelle ich nachfolgende Tabelle auf und bezeichne in derselben mit:

$V_{w}$ den Massenvorrat der ordentlichen Abnutzungsfläche,

$V_{u}$ den Sollvorrat dieser Fläche im Alter $u$,

$W_{w}$ den Massenvorrat der außerordentlichen Abnutzungsfläche, $W_{u}$ den Sollvorrat dieser Fläche im Alter u:

(Siehe Tabelle S. 18).

Die Größen $\mathrm{V}_{\mathrm{u}}$ und $\mathrm{W}_{\mathrm{u}-20}$, also die Sollvorräte für die Alter u und $u-20$, lassen sich aus den durch stammweise Aufnahme erhobenen Hat-Vorräten $\nabla_{\mathbb{w}}$ und $W_{w}$ der zu nutzenden Bestände mit Hilfe der Ertragstafeln annähernd ableiten. Übrigens läßt sich auch der Durchschnittszuwachs zu den betreffenden Feststellungen rerwenden, 


\begin{tabular}{c|c|c}
\hline Von der festgestellten & Vom erhobenen & Vom \\
Abnutzungsfläche & Massenvorrat & Geldertrag \\
(in \% der Ertragsfläche) & der Abnutzungsfläche & des Massenvorrats + Zuw. \\
\hline
\end{tabular}

entfälit auf

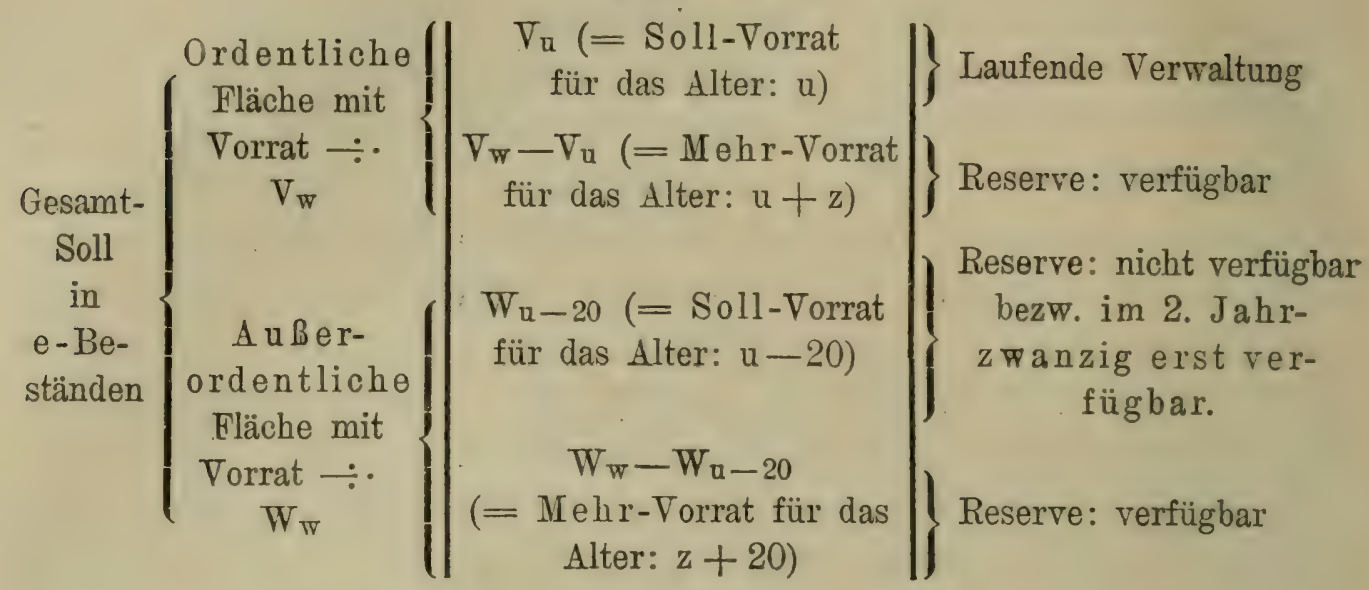

wenn die Größen $\mathrm{V}_{\mathrm{w}}, \mathrm{W}_{\mathrm{w}}$ und a (Alter) gegeben sind. Der Reserve geht hier ein Vorratsüberschuß zu von:

$$
=\mathrm{V}_{\mathrm{w}}+\mathrm{W}_{\mathrm{u}}-\left(\mathrm{V}_{\mathrm{u}}+\mathrm{W}_{\mathrm{u}-20}\right)
$$

doch besteht der oben hervorgehobene Unterschied in der Qualität der einzelnen Reserveteile.

b) Bei Flächenabmangel der Althölzer (wenn $\mathrm{F}_{\mathrm{e}}<\mathrm{F}_{\mathrm{n}}$ ).

Bei Abmangel in e +- -Beständen und teilweiser Ergänzung desselben aus d-Beständen setzt sich der nutzbare Massenvorrat zusammen aus:

a) e + -Beständen mit Überalter bezw. Vorratsüberschuß,

$\beta)$ d-Beständen mit Unteralter bezw. Vorratsabmangel.

Vom Vorratsüberschuß im Fall $\propto$ kann aber nur derjenige Teil als verfügbare Reserve angesehen werden, welcher nicht zur Deckung des Vorratsabmangels der d-Bestände erforderlich wird.

In der Regel wird der Mehrvorrat in e-Beständen größer sein als der Vorratsabmangel der d-Bestände, so daß auch im Fall des Flächenabmangels der Althölzer doch noch eine verfügbare Reserve sich ergibt.

Tabellarisch gestalten sich die Abnutzungsverhältnisse in der bei lit. a verwendeten Darstellungsweise folgendermaßen: 


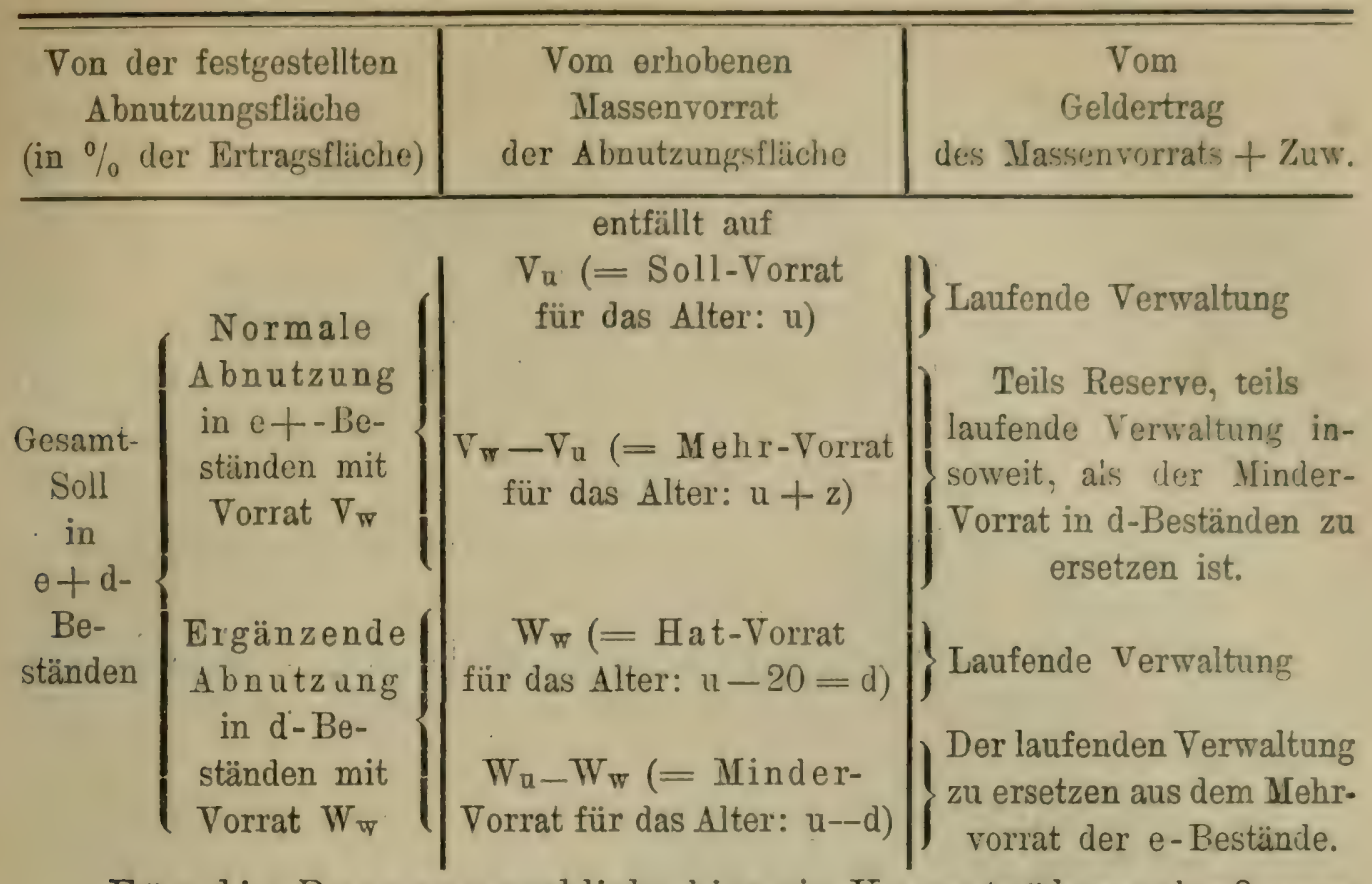

Für die Reserve verbliebe hier ein Vorratsüberschuß von: $=\mathrm{V}_{\mathrm{w}}+\mathrm{W}_{\mathrm{w}}-\left(\mathrm{V}_{\mathrm{u}}+\mathrm{W}_{\mathrm{u}}\right)$.

\section{Fall des Unteralters der Abnutzungs-Bestände}

\section{(Alter $\mathrm{a}=\mathrm{u}-\mathrm{z}_{\mathrm{l}}$ )}

a) Bei Flächenüberschuß der Althölzer $\left(F_{0}+>F_{n}\right)$.

Hier wäre zunächst die Frage zu entscheiden, ob das Unteralter vom finanziellen und waldbaulichen Standpunkt aus überhaupt zu berücksichtigen ist. Dieselbe wird verschieden $\mathrm{zu}$ beantworten sein nach Holzart und Standort der e-Bestände, auch nach dem Alter, insbesondere darnach, ob letzteres näher dem d-Alter oder der oberen Grenze vom u-Alter steht.

Ist die Differenz $u-z_{1}<10$, so dürfte der Fall des Unteralters unberücksichtigt bleiben, eine ordentliche Nutzung nach Maßgabe ron Ziff. 1 a oben anzusetzen sein. Die Folge wäre die, daß die ron $F_{0}$ übrig bleibenden Bestände in das 2. Jahrzwanzig übergehalten und nachgehends ein Überalter mit Vorratsüberschuß liefern würden.

Ist die Differenz $u-z_{1}>10$, so kann das Unteralter berücksichtigt werden in der Art, daß für den Vorratsabmangel der $\mathrm{F}_{\mathbb{w}}$ - Fläche eine entsprechende Ergänzungsnutzung durch Einstellung weiterer e-Bestände eingelegt wird. Das Maß dieser Ergänzungsnutzung bestimmt sich aus der Höhe des Vorratsabmangels der ordentlicherweise eingestellten Bestände bezw. aus der Differenz: $\mathrm{V}_{\mathrm{n}}-\mathrm{V}_{\mathrm{w}}$ und der dieser Differenz entsprechenden realen Nutzungsfläche. 
b) bei Flächenabmangel der Althölzer $\left(\mathrm{F}_{\mathrm{e}}<\mathrm{F}_{\mathrm{n}}\right)$ und $(\mathrm{a}<\mathrm{u})$.

Treffen beiderlei Abmängel zusammen: Flächen- und Vorratsabmangel, so muß in der Regel auf Ausgleichung verzichtet werden. Sie ist meist nur im Wege der Ermäßigung der Umtriebszeit zu erreichen und dann werden möglicherweise waldbauliche, auch finanzielle Gesichtspunkte $\mathrm{zu}$ sehr in den Hintergrund gedrängt werden.

Aus den unter Ziffer 1 und 2 angestellten Betrachtungen und den beigegebenen Tabellen geht folgendes herror:

a) Die Vorratsabnutzung läßt sich bezüglich Massen- und Geldertrag annähernd so regeln, daß unterschieden werden kann zwischen:

a) ordentlicher Kapitalnutzung, deren Geldertrag der laufenden Verwaltung zukommt und

ß) auBerordentlicher Kapitalnutzung bezw. Kapitalabtragung, deren Geldertrag einem Grundstock, einem Reservefonds, zuzuweisen ist;

b) die dem Grundstock zuzuweisenden Geldbeträge sind zweierlei Natur:

a) teils absolute Reserve: von eigentlichen Forratsüberschüssen herrührend und Ersparnisse früherer Wirtschaftsperioden darstellend, sofort verfügbar;

$\beta)$ teils relative Reserve: aus forstwirtschaftlich und finanziell derzeit gebotenen Nutzungen (Vorausnutzungen), deren Ertrag jedoch zur Deckung von Fehlbeträgen späterer Jahrzwanzig zu dienen hat; gebundene, zeitlich fixierte Reserve.

\section{Drittes Kapitel.}

\section{Die Organisation des Forsteinrichtungswesens.}

Bei jeder Einrichtung einer Betriobsklasse, welche Arbeit nit Aufstellung des Wirtschaftsplanes bezeichnet wird, siud nach den Ausführungen unter Kapitel I bis II zweierlei dufgaben zu lösen: eine umfangreiche forstliche Aufgabe, deren zweckmäßige und 
sichere Bewailtigung einerseits von geeigneten Kinrichtungsvorschriften, anderorscits ron wirksamer Organisation des ganzen Einrichtungswesens abhïngt, und eine fin anzielle A ufgabe, deren gnte be\%. praktisch brauchbare Lösung teils durch die Behandlung der forstlichen Aufgabe bedingt wird, teils durch die Sohaffung ciner gesctzlich festgelegrten Reservererwaltung, soweit es sich um Großbesitz, insbesondere denjenigen des Staates handelt.

Die Behandlung dieser Aifgaben wird praktisch darin zum Ausdruck liommen, daß am Schluß des Wirtschafteplans jeder Betriebsklasse eine Ausscheidung zwischen ordentliche: und auberordentlicher Flächen-bezw. Massennutzung vorgenommen wird und die Daten für Zuweisungen an die Resurvererwaltung, erent. mit Tremnung der Beträge in absolute und relative Reserve geliefert werden. Bei ciner VielzahJ ron Betriehsklassen, wie sie dem Staat und Großgrundbesitzer zur Verfügung stehen, ist es notwendig, daß jene Ausscheidung zwischen ordentlicher und auferordentlicher Nutzung in einheitlicher Weise rorgenommen und darauf die Organisation des Einrichtungswesens gegründet wird. Diese Organisation steht aber ihrerseits wieder mit derjenigen der gesamten Forstreiwaltung des Staates bezw. Grolggrundbesitzers in engem Zusammenhang.

Die allgemeinen Diensteseinrichtungen für die württembergischen Staatsforste sind gekeunzeichnet durch das reine Oberförstersystem mit Zentralisierung des Kontrolldienstes d. h. durch Gliederung der Verwaltung in Bezirksverwaltung und Aufsichtsführung durch eine Landesbehörde.

Die Bezirksverwaltung wird durch Forstänter besorgt. welche je nach der Bedeutung der Tirtschaftsobjekte teils nur mit einem Oberförster besetzt sind, teils außer diesem entwedor noch einen zweiten ständigen Beamten - Forstamtmann - haben oder rorübergehend über die geschäftsreichste Zeit des Jabres einen Forstassessor.

Der A ufsichts di enst wird unmittelbar durch die Forstinspektoren (Forsträte bezw. Oberforsträte), mittelbar durch die Forstclirektion ausgeübt, in welcher diese Inspektoren zu einem Landeskollegium unter Vorsitz eines fachmämnischen Direktors und in Unterordnung unter das Königl. Finanzministerium zusammengezogen sind. Diesem Kollegium gehört außer den Forstinspektoren (zurzeit 12) ein kameralistischer Verwaltungsreferent, ein Justitiar und der Wegbaureferent an. 
Zur Erledigung der Geschäfte hat die Direktion die erforderlichen Bureaus: neben einem allgemeinen Bureau für Besorgung der Schreib- und Revisionsgeschäfte ein Holzverkaufsbureau, ein Vermessungsbureau, ein wegbautechnisches Bureau. Von den Bureaus interessiert uns hier besonders das Vermessungsbureau wegen seiner Beziehung zur Forsteinrichtung. Dasselbe hat an Personal derzeit 4 Berufsgeometer, 2 Forstassessoren, 1 Kartenzeichner und 1 Lithographen. Seine Aufgave ist die Vorbereitung der Forsteinrichtung durch Vermessung noch nicht kartierter, insbesondere neu gebauter Wege, von Flächenänderungen infolge von Rechtsgeschäften (Kauf, Tausch), ferner durch die Ergänzung der Flurkartensammlungen (Katasterkarten) der Forstämter, die Erneverung der Flächenverzeichnisse als Besitzstandsdokumente, die Herstellung neuer Wirtschaftskarten, soweit erforderlich, und von farbigen Bestandeskarten, ausnahmsweise auch durch die Übertragung einer Neueinteilung des Waldes von den Karten ins Gelände u. a.

Die Oberaufsicht über dieses Vermessungsbureau wird durch einen der Forstinspektoren als Sachreferenten geführt, die gewöhnliche Bureauaufsicht durch einen hiermit beauftragten Geometer.

Die sonstigen Hilfsbeamten für Forsteinrichtung - Assessoren stehen mit dem Vermessungsbureau nicht in Zusammenhang. Sie wurden seither nach Bedarf den Forstämtern zugeteilt, in welchen Wirtschaftseinrichtungen stattfanden.

Der Wirtschaftsplan für die Staatswaldungen eines Bezirks wird bezw. wurde durch den Oberförster unter Leitung des Forstinspektors und in der Regel mit Unterstützung eines Assessors aufgestellt, nachdem die allgemein kartographischen und flächenstatistischen Grundlagen rom Vermessungsbureau geliefert waren.

Die Ergebnisse dieses Verfahrens der Aufstellung vou Wirtschaftsplänen haben nun - wir dürfen uns dies nicht rerhehlen in früheren Jahren den jetzigen Anforderungen an Einheitlichkeit der Behandlung, Sicherheit der Grundlagen und an das Tempo der Erledigung nicht immer entsprochen, werden noch weniger entsprechen, wenn den Forderungen der Kapitel I und II Rechnung getragen werden will. Wir sind daher zu ernstlicher Prüfung der Frage veranlaßt:

Wie ist in Anlehnung an diebestehende Organisation des allgemeinen Dienstes diejenige der Forsteinrichtung in zweckmäßiger d. h. wirksamer Weise zu regeln?

Zur Erörterung dieser Frage empfiehlt es sich, zunächst die Auf- 
gaben der forsteinrichtung kurz zu skizzieren. Dieselben bestehen in:

1. Lösung allgemeiner ökonomischer Fragen, insbesondere derjenigen über die Höhe der Umtriebszeit für die gegebenen Holzarten und Standorte.

Diese Frage ist meist für eine Gruppe von Bezirken, für hestimmte Wachstumsgebiete und mehr in der Instanz der Direktivbehörde zu behandeln, so daß sie als regelmäßige Aufgabe des Forsteinrichters in einem bestimmten Bezirk zunïchst ausscheidet.

2. Feststellung der Betriebsgrundlagen: des Hat an Produktionsfaktoren d. h. Ermittlung der Fläche nach Grölie: natürlicher und künstlicher Einteilung, welch letztere unter Umständen zu ändern ist, sodann nach Produktionsfähigkeit (Boden- und Inlturzustand), weiterhin Ermittlung der auf der Fläche und ihren einzelnen Teilen, den sogenannten Unterabteilungen befindlichen Holzbestände nach verschiedenen Gesichtspunkten wie Bestandesform, Holzarten, Alter, Schlußgrad, Geschichte, Holzrorrat in einem bestimmten Teil.

3. Feststellung der Betriebsrorschriften für die Zukunft: des Soll an Nutzung, Pflege und Kultur nach Zeit und Ort: d. h. für jeden Bestand bezw. jede Unterabteilung ist die wirtschaftliche Behandlung im kommenden Jahrzwanzig bezw. Jahrzehnt, die daraus sich ergebende Nutzung und zuwachspflegliche Maßnahme bezw. Wiederkultur zu bestimmen, und hierbei den Ansprüchen der Nachhaltigkeit, der Rentabilität, des Waldbaues, Forstschutzes und der Forstbenutzung Rechnung zu tragen.

Bei jeder Einrichtung kehren die Aufgaben 2 und 3 wieder und ich wende mich diesen im einzelnen zu.

\section{Die Ermittlung der Produktionsfaktoren (Inrentar- aufnahme).}

Die betreffenden Ermittlungen stellen gleichsam eine Inventaraufnahme nach Fläche, Boden und Bestand, getrennt nach den durch die natürliche und die künstliche forstliche Einteilung entstandenen sogenannten Distrikten, Abteilungen und Unterabteilungen (Einzelbestände im engeren Sinn) dar.

Diese Aufnahme ist, abgesehen ron den Arbciten etwaiger Änderung der Waldeinteilung und vorausgesetzt, daß einheitliche Grundsätze für diesolbe aufgestellt sind, eine mehr mechanische Arbeit, welche zwar nach jenen Grundsützen in systematischer 
Weise zu lösen ist, aber der örtlichen Erfahrung nicht bedarf, wie auch nicht größerer Reife des Urteils.

Die Aufnahmearbeit kommt zu hinsichtlich:

a) Vermessung und Kartierung des Besitzstandes bezw. von dessen Änderungen nach den Vorschriften über Fortführung der Landesvermessung, soweit erforderlich, dem Berufsgeometer und hat zu liefern:

a) das auf den öffentlichen Vermessungsakten beruhende Flächenverzeichnis, getrennt nach Markungen, Parzellen- und Wegnummern und mit Ausscheidung von ertragsfähigem Holzgrund, nicht ertragsfähiger Fläche und Nebengrund;

ß) Ergänzung der Flurkarten (Katasterkarten) auf den neuesten Stand nach den Ergänzungskarten der Gemeinden und den eigenen Messungen;

$\gamma)$ eine Übersichtskarte (Wirtschaftskarte) im Maßstab 1:20000 auf Grund der ergänzten Flurkarten.

b) Aufnahme des wirtschaftlichen Zustandes, seither auch Tatbestand genannt, dem Forsttechniker und hat zu liefern:

a) Die Festlegung und Aufnahme der forstlichen Einteilung d. h. Aussteckung von Abteilungslinien, Ausscheidung der Unterabteilungen, Vermessung der letzteren einschließlich Kartierung und Flächenberechnung;

B) Standorts - und Bestandesbeschreibung mit Bestimmung von Alter und Bonität;

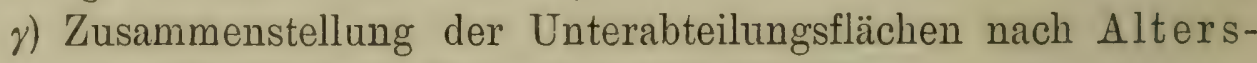
klassen, weiterhin nach Holzarten und Bonitäten;

d) Aufnahme der Holzvorräte in der ältesten Altersklasse bezw. in den für die Nutzung des nächsten Jahrzwanzig in Betracht kommenden Beständen;

ع) Zuwachsuntersuchungen usw. in den vorgenannten und weiteren Beständen;

$\zeta$ endlich eine Bestandeskarte mit farbiger Darstellung der Holzarten- und Altersklassenverteilung.

Es fragt sich nun, wer soll der Forsttechniker für diese Detailaufnahme, die Feststellung ron Größe und wirtschaftlichem Zustand von Unterabteilung zu Unterabteilung sein?

In dieser Hinsicht sind folgende Punkte ron Bedeutung:

1. Die Inventur hat in einheitlicher Weise für das ganze Land zu erfolgen und damit nach einem Arbeitsplan, wie auch der 
Geometer nach einer bindenden techischen Anweisung zu arbeiten hat. Auf diese Weise entstehen nicht nur gute Wirtschaftsplüne, sondern es werden auch überall die Produktionsgrundlagen in gleich, genauer Weise erhoben, so daß durch Zusammenstellung der Ergebnisse Unterlagen für Beurteilung wichtiger Fragen gewonnen werden.

2. Die Aufnahmearbeiten dürfen nicht durch anderweitige Dienstgeschäfte unterbrochen werden, müssen im Interesse der Einheitlichkeit, der richtigen Arbeitsteilung und der raschen Förderung in einem Zug erledigt werden.

3. Für die Arbeiten eignen sich nur jüngere, rüstige Kräfte, wclche längere Zeit auf diesem Gebiet tätig sind, und sich zu Spezialisten ausbilden, was nicht nur für die Förderung des Geschäfts, sondern für die Fortbildung der Aufnahme-Verfahren wichtig ist.

Diese Gesichtspunkte weisen zwingend darauf hin, die Detailaufnahme nicht den Lokalbeamten: Oberförster, eventuell Forstamtmann oder Forstassessor als außerordentliche Arbeit zuzuweisen, sondern besondere jüngere Forsttechniker zu bestellen, welche in der Detailaufnahme ausgebildet, diese als ihre ordentliche Arbeit besorgen und dem Forstamt ausschließlich für diesen Zweck beigegeben werden. Dadurch soll natürlich nicht ausgeschlossen werden, daß der Oberförster seine Lokalkenntnis und Anschauungen bei der Aufnahme, insbesondere bei der Ausscheidung der Unterabteilungen geltend macht, aber immerhin soll derselbe von diesen Aufnahmearbeiten tunlichst entlastet sein, um sich um so eifriger der 2. Hauptaufgabe der Einrichtung: dem Entwurf der Betriebsrorschriften, widmen zu können.

Als Einrichtungstechniker kommen Forstassessoren (Einrichtungsassessoren) in Betracht, welche, um eine Einheitlichkeit der Aufnahmen zu erzielen, nicht nur eines Arbeitsplanes, sondern auch des Zusammenhangs mit einer Aufsichtsbehörde und der Kontrolle bedürfen.

Wird nun schon bisher der eine Teil der Inventaraufnahme, der rein geometrische, durch Berufsgeometer der Forstdirektion besorgt, welche in einem Vermessungsbureau rereinigt und einem Sachreferenten unterstellt sind, so ist es meines Erachtens das Gegebene, jenem Bureau auch die Beamten für den forstlichen Teil der Iriventaraufnahme zuzuteilen und diese Beamten von der Zentralstelle des Bureaus aus nach Bedarf in den 
Forstbezirken des Landes zu rerwenden. Hiermit kämen wir a uf Erweiterung des bestehenden Vermessungsbureaus durch Zuteilung der erforderlichen Anzahl von Forstassessoren zu einem Vermessungs- und Einrichtungsbureau, welches der Kürze wegen "Einrichtungsbureau der Forstdirektion" mit Anlehnung an die Bezeichnung der übrigen Bureaus dieser Behörde genannt werden kann.

Die Einrichtungsassessoren sind für ihre Aufgabe durch einen geodätischen Kürs \%u schulen, erhalten für jedes Jahr je nach dem Stand der Einrichtungen eine bestimmte Marschroute, haben nach einem Geschäftsplan zu arbeiten und einen Bezirk vor Abschluß der Einrichtungsarbeiten nicht zu verlassen. Eine wenn auch nur vorübergehende Verwendung im laufenden Dienst des Forstamts soll untersagt sein.

Die Schulung der Assessoren für ihre Aufgabe, die Einleitung und Kontrolle der Arbeiten, die Fürsorge für einheitliche Durchführung des Arbeitsplanes ist einem erfahrenen Beamten ständiger Art, welcher auch dem Einrichtungsbureau zuzuteilen wäre, zu übertragen, einem Forstamtmann. Dieser Beamte sollte womöglich schon früher als Assessor bei der Einrichtung tätig gewesen sein.

Ein Amtmann genügt meines Erachtens für die betreffende Tätigkeit, empfiehlt sich auch mit Rücksicht auf körperliche Leistungsfähigkeit und mehr als eir Oberförster, der in Jahren schon rorgerückt ist und nach mehrjähriger Verwaltung eines Forstbezirlies sich bei der vorherrschenden Bureaubeschäftigung kaum wohl und befriedigt fühlen wird.

Des weitern erfordert die Schaffung eines »Einrichtungsbureaus * in obigem Sinn nicht nur im Hinblick auf die Flächenaufnahmen, sondern noch mehr für Erhebung der Bestandscharaktere, der unter lit. b genannten Produlitionsfaktoren, sowie der Holzrorräte und Zuwachsgrößen die Beigabe ron Meßgehilfen für jeden Aufnahmebeamten. Als solche Gehilfen, welche ron den Assessoren in Zeitkürze eingelernt werden können, empfehlen sich die fachlich einigermaßen vorgebildeten Anwärter des Forstschutzdienstes, die Forstanwärter, welche nicht nur den Vorzug einiger Waldkenntnisse hätten, sondern auch in cinem gewissen Unterordnungsrerhältnis zu den Aufnahmebeamten, wie zu dem betreffenden Forstamt stünden. Diese Gehilfen liönnen bei Unterbrechungen der Aufnahmen im Wald, bei anhaltendem Regenwetter zu den zahlreichen mechanischen Berechuungen rerwendet werden, welche die Aufnahmen im 
Gefolge haben. Hierdurch würden sie auch für ihren künftigen Beruf mehr eingeschult.

Ein wesentlicher Mehraufwand durch die Verwendung der Gehilfen entstünde nicht, da andernfalls an Ort und Stelle Meßgehilfen ron meist zweifelhafter Brauchbarkeit gewonnen werden müssen. Übrigens sind auch schon bisher da und dort Forstanwärter zu cinem Teil der Inventarisierung, zu den Holzvorratsaufnahmen mit Erfolg verwendet worden, ${ }^{1}$ ) und könnten auch für die Zukunft rom Kommando der Forstwache nach Bedarf zur Verfügung gestellt werden.

Durch die rorgeschlagene Ausgestaltung des Einrichtungsbureaus würde zugleich eine Art Spezialistentum für forstliche Flächen- und Massenaufnahmen geschaffen, welches zu Verbesserungen und Vereinfachungen besonders auch auf dem Gebiet der Vorratsaufnahmen. der Zuwachs- und Ertragsberechnungen führen wird. Gerade die Zentralisierung der Massenaufnahmen, bei welchen nicht immer sorgfaltig genug rorgegangen wird, ihre einheitliche und sichere Gestaltung haite ich, namentlich auch im Hinblick auf die Schaffung ron Lokal-Ertragstafeln, schon längst für ein Bedürfnis.

\section{Die Feststellung rom Soll an Nutzung, Pflege und Kultur.}

Liegen die Ergebnisse der Inventaraufnahme durch das Einrichtungsbureau vor, welche Aufnahme rielfach zu den Torarbeiten der Eimrichtung gerechnet wird, so schließen sich die Hauptarbeiten der letzteren an: die Disposition über das erhobene Inrentar, der Entwurf der Betriebsrorschriften, rum Soll der Wirtschaft und der Nutzung für das nächste Jahrzwanzig bezw. Jahrzehnt ron Unterabteilung zu Unterabteilung. In der Summierung der Sollbeträge für die letzteren ergibt sich das Soll der Betriebslilasse an Hauptnutzung, Zwischennutzung, Kulturen.

Zur Lösung dieser Aufgabe ist örtliche Erfahrung und eine gewisse Reife des Urteils ron großer Bedeutung, weshalb ihre Behandlung meines Erachtens in erster Linie dem Oberförster in Verbindung mit dem Forstinspektor, dessen Orts- und Sach-

1) Ich halue hier eine :̈hnliche Einrichtung im Auge, wie sie bei unserer forstlichen Versuchsanstalt Tübingen schon seit deren Gründung besteht. die Einrichtung, daß dem Aufuahmeassistenten für dic Dauer der Aufnahmen im Wald ein ständiger Gehilfe beigegeben ist, welcher jeden Ortswechsel mitmacht. Ein solcher Wechsel wäre jedoch bei der Forsteinrichtung selten nötig. 
kenntnis sich über ein noch größeres. meist verwandtes Gebiet erstreckt und welcher in der Regel über reichere Erfahrung verfügt, zukommt.

Es hieße die Sache, wie die Berufsfreude des Oberförsters schädigen, wenn nicht dessen Ortskenntnis und Erfahrung in weitestem MIaß ausgenützt würde! Alles Wissen und alles Können des Oberförsters soll im Wirtschaftsplan auskristallisieren: dieser soll ein Denkstein seiner Tätigkeit sein, wenn auch formell nur für ein Jahrzehnt, aber materiell auf ein oder mehrere Jahrzwanzig fortwirkend. Sodann liegt ein innerer Widerspruch vor, wenn der Verwaltungsbeamte, welcher den Wirtschaftsplan auszuführen hat, nicht auch bei dessen Aufstellung in entsprechendem Maß mitwirkt und diese grundlegende Arbeit der Wirtschaft jüngeren Beamten verbleibt, also den letzteren die schwierigere Arbeit, dem älteren und gereiften Lokalbeamten die einfachere Aufgabe der Ausführung des Planes.

Die intensive Mitwirkung des Oberförsters erscheint um so mehr möglich und geboten, wenn ihm die mechanischen Arbeiten der Inventur von Fläche und Bestand nach Ziffer 1 abgenommen werden, weiterhin wenn, was für Württemberg zutrifft, die Forstbezirke eine angemessene Durchschnittsgröße haben d. h. nicht zu groß sind und auch aus diesem Grund der Vielgestaltigkeit der Waldverhältnisse gebührend Rechnung getragen werden kann.

Analoge Gründe, wie für die Betätigung des Oberförsters bei den Arbeiten zu Ziffer 2 sprechen für diejenige des Forstinspektors. Dieser ist der weitere Träger der örtlichen Erfahrung, aber auch der Erfahrung aus größerem Gebiet, der umfassenderen Geschäftskenntnis bei der großen Zahl von Wirtschaftsplänen, bei welchen er mitzuwirken hat. Sodann spricht zugunsten der Mitarbeit des Forstinspektors die Rücksicht darauf, daß demselben die Prüfung: der jährlichen Betriebspläne obliegt und solche um so sachgemäßer erledigt werden wird, wenn der Beamte auch bei Aufstellung des Hauptbetriebsplans fürs Jahrzehnt, des Wirtschaftsplans, mitgewirkt hat. Diese Mitwirkung sehe ich als einen wesentlichen Bestandteil der Aufgabe der Forstinspektoren an! Die Ausschaltung der letzteren und Ersatz ihrer Arbeit durch den Beamten einer geschlossenen Einrichtungsanstalt, welcher sich durch wenige Besuche des Bezirkes kaum genügend $z u$ orientieren vermöchte, erschieneu mir eincrseits als ein sachlich folgenschwerer Fehler, andererseits als ein Eingriff in unsere Dienstorganisation überhaupt.

Hiernach steht es mir außer Zweifel, daß bei den Hauptarbeiten 
der Einrichtung (Ziff. 2) zunächst Oberförster und Forstinspektoren teils entwerfend, teils prüfend zusammen zu wirken haben.

Diese Hauptarbeiten gliedern sich folgendermaßen:

a) Entwurf der Betriebsvorschriften - fürs nächste Jahrzwanzig, was die Hauptnutzung anbelangt, fürs nächste Jahrzehnt bezüglich der Zwischennutzungen: Durchforstungen und Reinigungen, sowie der Kulturen;

b) provisorische Feststellung der Abnutzungsfläche für die nach lit. a zur Nutzung im nächsten Jahrzwanzig rorzusehenden oder möglichen Bestände im einzelnen und im ganzen, des Flächenplans der Hauptnutzung, weiterhin auf Grund hiervon, der Vorrats- und Ertragsberechnungen des Massenplans dieser Nutzung;

c) Feststellung des Flächenplans der Z wischennutzungen bezw. der zuwachspfleglichen Maßnahmen an Durchforstungen und Reinigungen, mit Veranschlagung des mutmaßlichen Ertrags der Durchforstungen;

d) Bestimmung der Kulturflächen, des sogenannten periodischen Kulturplans.

Die sämtlichen Angaben zu lit. a-d sind in der Reihenfolge der Distrikte und Abteilungen, event. Hiebszüge je nach Unterabteilungen und in Gesamtsumme zu liefern.

Wie ersichtlich ist die Arbeit zu lit. b, die Feststellung der Abnutzungsfläche zunächst provisorisch, d. h. im Entwurf von Oberförster und Forstinspektor zu fertigen. Zur endgültigen Bestimmung jener Fläche erachte ich die Mitwirkung eines weiteren Faktors, des Sachreferenten rom Einrichtungsbureau der Forstdirektion für wünschenswert oder im Hinblick auf Kapitel I und II meiner Ausführungen für geboten. Die Regelung der Abnutzungsfläche hat einerseits für das ganze Land nach gewissen einheitlichen Grundsätzen zu erfolgen, für deren Einhaltung der Sachreferent sorgt und hierin eine gewisse Kontrolle ausuibt, andererseits ist sie von Bedeutung für die Ausscheidung der Erträge, welche der laufenden Verwaltung zukommen und welche einer Reserveverwaltung. Es ist dies ein neues Moment, welches in die Beurteilung der ganzen Frage hereinkommt.

Gegebenenfalls wäre der Entwurf zu lit. b dem Sachreferenten der Forstdirektion zu übergeben, von diesem zu prüfen und im Benehmen mit Forstinspektor und Oberförster endgültig festzustelleu. Bei Meinungsverschiedenheit der Beteiligten hätte die Forstdirektion 
zu entscheiden. Ist der bezeichnete Entwurf genehmigt, so steht dem weiteren Fortgang der Arbeiten zu lit. c und d nichts mehr im Weg.

Den Abschluß der Arbeiten bildet die Zusammenfassung sämtlicher Ergebnisse der Einrichtung, besonders auch der wirtschaftlichen Grundsëtze für die Zukunft im „Einrichtungsprotokoll“, für welches sich ein einheitliches Schema empfiehlt, was Rubrikenordnung und Tabellenbeigabe betrifft. Bei der Abfassung dieses Protokolls hätten auch wieder Oberförster und Forstinspektor zusammenzuwirken.

Geschäftlich betrachtet zerfallen hiernach die Hauptarbeiten zu Ziffer 2 in 3 Abschnitte:

a) die Arbeiten bis zum Entwurf des Flächenplans der Hauptnutzungen (der Abnutzungsfläche) durch Oberförster und Forstinspektor;

b) Prüfung der von diesen beantragten Abnutzungsfläche durch den Sachreferenten der Forstdirektion und endgültige Feststellung derselben im Benehmen mit Oberförster und Forstinspektor, event. mit Ausscheidung der zur ordentlichen Abnutzung vorgesehenen Flächen und der zur außerordentlichen Nutzung kommenden Flächen;

c) die übrigen Arbeiten: Ertragsberechnung und Nutzungsfestsetzung fürs nächste Jahrzwanzig bezw. Jahrzehnt, event. auch hier mit Trennung der der laufenden Verwaltung und der einer Reserveverwaltung zugehenden Erträge, Abfassung des Einrichtungsprotokolls je durch Oberförster und Forstinspektor.

Wurden unter Ziffer 1 und 2 die Arbeiten erörtert, welche bei Aufstellung bezw. Erneuerung eines jeden Wirtschaftsplanes wiederkebren, weiterhin die geeigneten Organe für Erledigung der Arbeiten benannt, so werde ich unter Ziffer 3 die Ausgestaltung des rorgeschlagenen Einrichtungsbureaus im einzelnen, seinen Zusammenhang mit dem Sachreferenten und dessen Aufgabe behandeln.

\section{Die Organisation von Einrichtungsreferat and Ein- richtungsbureau im besonderen.}

Bei Bewältigung der umfassenden Einrichtungsarbeiten ist genaue Umgrenzung der Zuständigkeiten der nach dem vorgetragenen erforderlichen Organe sowohl in sachlicher als in persönlicher Hinsicht von Bedeutung, sodann die gesamte Organisation der Arbeit mit Rücksicht auf die Kosten.

Als besondere Organe wurden benannt: 


\section{A. Der Sachreferent der Forstdirektion.}

Seine $\Lambda$ ufgabe kann dahin präzisiert werden: or hat zu besorgen :

a) die Behandlung allgemeiner ökonomischer Fragen insbesondere über Bestimmung der Umtriebszciten für die einzelnen Wachstumsgebiete nach Holzart, Standort usw., also der Fragen, welche eingangs als erste Hauptaufgabe der Forsteinrichtung im allgemeinen, als eine Art Vorfrage bezeichnet worden ist;

b) die Fortbildung des Einrichtungsverfahrens, welche sich in fortlaufender Verbesserung der Einrichtungsrorschriften, derzeit in Neubearbeitung der Einrichtungsinstruktion auszudrücken haben wird;

c) die Leitung des Einrichtungsbureaus, bestehend in Geschäftsverteilung und Geschäftsanweisung, welch letztere den Forstassessoren in Form eines "Arbeitsplanes" in die Hand zu geben ist, ferner die oberste Kontrolle des Geschäftsvollzugs;

d) die Mitwirkung bei Aufstellung des einzelnen Wirtschaftsplans insoweit, als es sich um wesentliche Einteilungsänderungen handelt, weiterhin um Prüfung und endgültige Feststellung (ỉer Abnutzungsfläche, event. um die Trennung zwischen ordentlicher und außerordentlicher Nutzung, je im Benehmen mit dem Forstinspektor und Oberförster;

e) die Ausbildung und Leitung derEinrichtungsstatistik, um das Material für Beurteilung entscheidender Fragen der Nachhaltigkeit, der Rentabilität usw. zu liefern.

\section{B. Das Einrichtungsbureau.}

Als Aufgaben dieses Bureaus wären zusammenfassend zu bezeichnen :

a) die Aufnahme des gesamten Inventars sowohl an Flïche nach rechtlichem Besitzstand, forstiicher Einteilung und Bodenbeschaffenheit, als an Waldbestand nach seiner Zusammensetzung und seinen charakteristischen Größen;

b) die kartographische und tabellarische Darstellung der Aufnahmeergebnisse nach Maßgabe der hierauf bezüglichen Vorschriften;

c) die A usbildung der für die Inventaraufnahme erforderlichen forstlichen Hilfsbeamten, in erster Linie der Aufnahmeassessoren und durch dieser der Meßgehilfen;

d) die Einleitung der Aufnahmearbeiten in den einzurichtenden Wirtschaftsbezirken und Ausstattung des Personals mit den nötigen 
Instrumenten, Rechnungshilfen usw., weiterhin die Fürsorge für ungestörten Fortgang der Arbeiten;

e) die Prüfung aller mit der Disposition über das Inventar zusammenhängenden Berechnungen in den Wirtschaftsplänen;

f) die Bearbeitung der Einrichtungsstatistik.

Für Erfüllung dieser Aufgaben würden in Württemberg zunächst zwecks Bearbeitung der Staatswaldwirtschaftspläne, zu bestellen sein:

1. ein Forstamtmann (event. Oberförster) als Geschäftsführer unter Leitung des Sachreferenten und als Bureauvorstand;

2. Einrichtungs-Assessoren, vorläufig 15, in der Hauptsache für Inventaraufnahme und deren Bearbeitung, ron welchen 3 ständig sich beim Bureau befänden für kartographische und andere Arbeiten, 12 unständig d. h. für die bessere Jahreszeit, etwa rom 1. April bis 1. November, und für die Aufnahmen im Wald zugeteilt würden;

3. Meßgehilfen für jeden Aufnahmeassessor in Gestalt eines Forstanwärters, welcher bei den Flächenaufnahmen und -Berechnungen mithilft und die Massenaufuahmen, event. unter Zuziehung der Forstwarte besorgt.

Mehr in Form eines Zusatzes möchte ich noch die Einrichtungsarbeiten in Körperschaftswaldungen berühren, welche nach dem württ3mbergischen Körperschaftsforstgesetz dem Oberförster obliegen, abgesehen von den eigentlichen Vermessungsarbeiten. Im Interesse rascherer Förderung jener Arbeiten balte ich es für durchaus geboten, daß die Inventaraufnahme in solchen Waldungen, welche eine gewisse Größe überschreiten, auch ron dem staatlichen Einrichtungsbureau und zwar auf Kosten der betreffenden Körperschaften ausgeführt wird.

Die Flächenaufnahme der Unterabteilungen fällt meines Erachtens unter die geometrischen Arbeiten, welche nach $\S 5$ der Vollzugsverfügung zum Körperschaftsforstgesetz neben der Fertigung der Übersichtskarte einem öffentlichen Feldmesser zu übertragen sind. Die Übertragung an F'eldmesser hat jedoch das Bedenken, dah die Aufnahme mit einem Genauigkeitsgrad erfolgt, welcher der Bedeutung derselben nicht entspricht und erfahrungsgemäß zu hohe Kosten verursacht. Auch werden die Arbeiten selten rechtzeitig ausgeführt. Sachgemäßer und billiger für die Waldbesitzer wäre sicher die Übertragung an das staatliche Einrichtungsbureau gegen Ersatz der Kosten. 
Die schwierige Frage ist jedoch, von welcher Flächengrïße des Waldbesitzes an Übernahme der Vermessungsarbeiten durch das Bureau angezeigt wäre? Wird zunächst unterstellt, daß bei einer Waldfläche von -: 400 ha für eine Gemeinde jene Übernahme erwünscht ist, so kämen nach meiner Rechnung rund 75 von den in Staatsbeförsterung stehenden Körperschaften in Betracht, so daß rund 8 Einrichtungen auf das Jahr entfielen. Von den Körperschaften, welche durch eigene Sachverständige bewirtschaftet werden $(84 \mathrm{zu-}$ sammen), käme aber auch noch ein Teil hinzu und wollen hierfür 2 Einrichtungen fürs Jahr angesetzt werden. Fis ergäben sich hiernach 10 Einrichtungen im Jahr, ron welchen jedoch $2-3$ ron einem Assessor bewältigt werden könnten, so daß als weiteres Personal für Einrichtungsarbeiten in Körperschaftswaldungen durch das Bureau 4 unständige Assessoren in Aussicht zu nehmen wären.

Jedenfalls könnten durch ein Personal ron insgesamt 20 Assessoren beim staatlichen Einrichtungsbureau vorläufig alle Ansprüche ron Staats- und größeren Körperschaftswaldungen hinsichtlich der Inventaraufnahmen für Einrichtungszwecke befriedigt werden.

\section{Viertes Kapitel.}

\section{Die Reservebildung und ihre gesetzliche Regelung.}

\section{Die Reserve und ihre Bestimmung im allgemeinen.}

Die nach dem Eingang zu Kapitel III bei jeder Forsteinrichtung zu lösende finanzielle Aufgabe setzt einmal die richtige Erfüllung der vorausgegangenen forstlichen Aufgabe, sodann aber die Schaffung einer Reserveverwaltung voraus, deren finanzielle Bestimmung gesetzlich festzulegen ist. Wir haben dem Naturalfonds des Waldbetriebskapitals, welcher aus Boden, Hoizbestand, Bringungsanstalten usw. besteht und eine ordentliche laufende Materialnutzung, unter Umständen eine außerordentliche Materialnutzung abwirft, zur Seite zu stellen einen Geldfonds oder Geldgrundstock der Forste, in welchen die Gelderträge aus den außerordentlichen Nutzungen fließen. Entsprechend dem verschiedenen Charakter der letzteren Nutzungen wären in jenem Grundstock zunächst $2 \mathrm{Ab}$ teilungen zu bilden, welchen je besondere Bestimmung zukommt: 
Abteilung A für absolute oder freie Reserve, als Verfügungsfonds des Grundstocks zu bezeichnen, aus MaterialNacherhebungen, also Ersparnissen fr üherer Zeit herrührend, welche übrigens auch mit Ermäßigungen des Umtriebs bezw. des Betriebskapitals zusammenhängen können.

Abteilung B für relative oder gebundene Reserve, als Ausgleichsfonds des Grundstocks zu bezeichnen, aus MaterialVorerhebungen der Gegenwart auf Rechnung der Zukunft herrührend und nach Kapital samt zugewachsenen Zinsen erst in späteren Zeiten, frühestens zu Beginn des 2. Jahrzwanzig verfügbar.

Aus dieser Charakterisierung der Fondsabteilungen läßt sich deren Bestimmung folgendermaßen ableiten:

A. Der Verfügungsfonds des Grundstocks.

Ich setze voraus, daß sowohl Kapital als Zinsen zur weiteren Ausgestaltung des Naturalfonds als Produktionskapital und so zur direkten oder in direkten Hebung der Erträge der laufenden Verwaltung verwendet werden. Die Verwendung kann in dieser Absicht erfolgen:

a) zur Vergrößerung des Naturalfonds (Grundstocksmehrung) durch Erwerbung (Kauf, Tausch usw.),

b) zur Verbesserung des Naturalfonds (Grundstocksverbesserung) durch

a) Entlastung des Waldes von finanziell und rolkswirtschaftlich schädlichen Holzgerechtigkeiten,

$\beta$ ) Verbesserung und Mehrung der Absatzwege (Bau von Wegen, Eisenbahnen usw.), um durch Erhöhung der Erlöse diejenige der laufenden Verwaltung herbeizuführen;

r) Verbesserung der Methoden der Bestandesbegrïndung und -Ernte, was geschieht durch Anschaffung nener geeigneter Werkzeuge und Vorrichtungen, Anstellung von Versuchen usw.;

d) Verbesserung der Verwaltungseinrichtungen;

c) zur Sicherung des Naturalfonds (Grundstockssicherung) vermittelst Verbesserung der Forstschutzeinrichtungen einschließlich der Erstellung von Forstwarthäusern, des Beitritts zu einer Feuerversicherung, ohne übrigens hier der Versicherung des Staatswaldbesitzes das Wort reden zu wollen.

B. Der Ausgleichsfonds des Grundstocks.

Diesem kommt nur die eine Hauptbestimmung zu, nach Ablauf eines durch den Wirtschaftsplan festzusetzenden Zeitraumes, in der 
Regel eines Jahrzwanzig, während welchen Zeitraumes die Zinsen dem Kapital unvermindert zuzuwachsen haben, Zuschüsse zur laufenden Verwaltung jener späteren Zeit zu liefern.

Die Geldzuschüsse sind bedingt durch Abnormität des Altersklassenverhältnisses im Zusammenhang mit dem Bestreben, überalto Bestände finanziell rechtzeitig abzunutzen. Dor hierdurch verursachte Ausfall an späterer Materialnutzung wird durch Geldbezug aus dem Grundstock ersetzt bezw. ausgeglichen.

Den unter lit. A und B behandelten Fondsabteilungen kommt Grundstocksnatur zu. Ihr Zufluß wird durch die Forsteinrichtung nach Kapitel I und II geregelt. Es ergibt sich jedoch für den laufenden Betrieb ebenfalls Anlaß zu Fondsbildung, um den Schwankungen des Holzmarktes Rechnung tragen zu kïnnen, welclen Fall ich in lit. C betrachten möchte.

\section{Der A usgleichsfonds der laufenden Verwaltung.}

Durch die Ertragsregelung bei der Forsteinrichtung wird zunächst die Erhebung jährlich gleicher Nutzungen vorgesehen, ebenso bei Aufstellung des Forstetats für die Zwecke des Hauptfinanzetats eine im roraus festgesetzte Nutzungsgröße, wie ja hier auch der Einheitspreis bezw. der gesamte Geldertrag im roraus reranschlagt werden muß. Im Lauf der Erhebung der Nutzungen zeigen sich nun mehr oder weniger erhebliche Abweichungen von den rorgesehenen Preisen: teils Steigerungen, welche Überschüsse der laufenden Verwaltung gegenüber den Voranschlägen zeitigen, teils Preisrückgänge, welche zu plötzlichen Preisstürzen ausarten künnen und Ertragsausfälle zur Folge haben. Hierbei sollten die Überschüsse der guten Jahre entschieden einer Fondsabteilung zufließen, damit aus denselben die Abmängel schlechter Jahre, insbesondere die Ausfälle infolge plötzlichen Preisrückgangs gedeckt werden können. Diese Fondsabteilung diente hiernach wesentlich zur Sicherung der statlichen Etatswirtschaft. Ein erheblicher Preisrückgang legt es des weiteren nahe, das ordentliche Nutzungsquantum eines Jahres vorbehältlich der $\mathrm{N}$ ach erhebung zu günstigerer Zeit absichtlich zu kürzen und in dem Reservefonds finanzielle Deckung für den weiteren Ertragsausfall zu suchen. Der Fonds sollte die Möglichkeit geben, daß eine Anleihe bei ihm gemacht und diese heimbezahlt würde, sobald die Holzpreise günstigere geworden sind und die Erbebung der eingesparten Nutzung nachgeholt werden kann. 
Andererseits sollten zur Ausnutzung einer Hochkonjunktur vorübergehende Mehrfällungen gestattet sein und die Zuweisung von deren Erträgen an die Reserve erfolgen. Entsprechende Kürzung der nachfolgenden Nutzung wäre hier erforderlich.

In diesen Fällen diente der Fonds dem $\mathrm{kaufmännischen}$ Betrieb der Forstverwaltung und ermöglichte es der letzteren, ron der Gleichheit der Materialnutzungen in den einzelnen Etatsjahren abzugehen, die Größe dieser Nutzungen der Marktlage anzupassen und hiemit auch den weiteren ('ang der Preise zu beeinflussen.

Hier fragt es sich nur, ob nicht für die Ausgleichung der erwähnten Weniger- oder Mehrnutzung ein bestimmter Zeitraum festgestellt werden soll, damit nicht zu große Unsicherheit in die Massenund Finanzwirtschaft kommt. Hierüber ist besondere Untersuchung geboten.

Jedenfalls müßte dieser Ausgleichsfonds der laufenden Verwaltung schon erheblich dotiert sein, um voll wirksam werden $\mathrm{zu}$ können.

Würden nach den Ausführungen unter $A-C \quad 3$ Abteilungen unterschieden, so wäre auch getrennte Buchführung für dieselben notwendig und die Reserveverwaltung stellte eine Art Bankinstitut dar, aus dessen Rechnungsstand jederzeit die Mn̈glichkeit und das $\mathrm{Maß}$ von Aufwendungen für einen der bezeichneten Zwecke beurteilt werden könnte.

\section{Das bestehende Württembergische Reservefondsgesetz.}

Das württembergische Gesetz betreffend Einrichtung eines Reservefonds der Staatsforsten vom 1. August 1905 (Reg.-Bl. S. 149) lautet:

,Artikel 1.

Das Finanzministerium wird ermächtigt, innerhalb der Gültigkeitsdauer dieses Gesetzes außerordentliche Holznutzungen in den Staatswaldungen bis zum Gesamtbetrage von $300000 \mathrm{fm}$ Derbholz und die dadurch bedingten Ausgaben für Holzhauerlöhne, Wegbanten und Kulturen anzuordnen. Die dusgaben für Wegbauten dürfen 700 M, die Ausgaben für Kulturen 500 M für je $1000 \mathrm{fm}$ Holznutzung nicht übersteigen.

Die aus den außerordentlichen Holzuntzungen hiernach sich ergebenden Reinerträge fließen in einen von der Staatshauptkasse zu verwaltenden Reservefonds der Staatsforsten, dessen Bestände verzinslich anzulegen sind. Die Zinsen kommen dem Reservefonds zu.

Größere infolge von Naturereignissen eintretende Holzanfälle sind, soweit sie nicht durch eine Ermäßigung der ordentlichen Nutzungen ausgeglichen werden können, mit ihrem Reinertrag dem Reservefonds zu überweisen und 
sollen den nach Abs. I zugelassenen außerordentlichen Nutzungen zugerechnet worden.

\section{Artikel 2.}

Die Mittel des Reservefonds dienen zur Deckung von Fehlleträgen, welche sich beim Reinertrag aus den Staatsforsten gegenüber dem verabschiedeten Hauptfinanzetat ergeben.

Zur Deckung von Fehlbeträgen soll innerhall der fültigheitsdauer des Gesetzes in jedem Etatsjahr beginnend mit dem Etatsjakr 1907 der Reinertrag einer Nutzung von $30000 \mathrm{fm}$ Derbholz, sofern diese vollzogen ist, samt den bis zum Schlusse des betreffenden Etatsjahres angefallenen Zinsen zur Verfügung gestellt werden. Der von dieser Summe in sinem Etatsjahre nicht erforderlich gewordene Betrag wächst je dem für das folgende Etatsjahr verfügbaren Reinertrag $z u$.

\section{Artikel 3.}

Das Finanzministerium wird ermächtigt, beim Eintritt eines erheblichen Preisrücligangs die ordentliche Nutzung unter den dem Voranschlag im Hauptfinanzetat zugrunde liegenden Betrag insoweit zu ermäßigen, als der durch diese Mindernutzung entstehende Ausfall aus den vorhandenen Beständen des Reservefonds unbeschadet der Erfüllung der dem letzteren in dem betreffenden Jahre nach Maßgabe des Art. 2 zufallenden Aufgabe gedecht werden laann.

Der dem Reservefonds hiernach entnommene Betrag ist demselben in künftigen Jahren wieder zu ersetzen, sobald die Mindernutzung im Tege uachträglicher Erhebung wieder ausgeglichen wird.

\section{Artikel 4.}

Die Bestimmungen dieses Gesetzes gelten bis zum 31. März 1917.

Nach Ablauf der Gültigkeitsdauer des Gesetzes wird über den etwa rorhandenen Bestand des Reservefonds durch Verabschiedung mit den Stïnden (Verf.-Urkunde \& 181) Verfügung getroffen werden.:

Die Bedeutung dieses Gesetzes, welches den Ausgangspunkt der Geldreservebildung für unsere Staatsforste darstellt und in gewissem Sinn einen Vorgang für andere Staaten mit großem Forstbesitz geschaffen hat, unterschätze ich keineswegs, aber im Hinblick auf die Ausführungen unter Ziffer 1, erscheint mir in dem Gesetz der Begriff der Reserve zu eng und grundsätzlich nicht ganz zutreffend gefaßt zu sein. Es wurde durch dasselbe nur ein Ausgleichsfonds der laufenden Verwaltung begründet, während außerdem weder ein Verfügungsfonds des Grundstocks, noch ein Ausgleichsfonds des letzteren geschaffen ist, und des weiteren wurde jener Ausgleichsfonds gefüllt mit den Erträgnissen ron Nutzungen, welche außerordentlicherweise erhoben worden sind und eigentlich dem Geldgrundstock der Staatsforste zuzuscheiden gewesen wären. 
Die Fondsmittel haben nach dem Gesetz in erster Linie zur Deckung von Fehlbeträgen, welche sich beim Reinertrag aus den Staatsforsten gegenüber dem verabschiedeten Hauptfinanzetat ergeben, zu dienen, also um Schwankungen der laufenden Gelderträge auszugleichen, in subsidiärer Wंeise dazu, un in Fall eines Preisrückganges die Nutzung ermäßigen und den hierdurch entstehenden Ausfall an Geldertrag aus dem Fonds vorschußweise decken zu können.

Wenn auch durch Abs. 2 von Art. 2 die Benützung des Fonds geregelt wurde, so ist derselbe doch beim ersten größeren Preisrückschlag von einiger Dauer der Abnutzung ausgesetzt.

Es werden dann Fehlbeträge gegenüber den Etatssummen mit Ermäßigung der Nutzung und Deckung des hieraus entstehenden Ausfalls zusammentreffen und den Fonds so schwächen, daß er seine Zwecke kaum mehr zu erfüllen vermag, geschweige denn, weiteren Verpflichtungen, wie sie aus der Grundstocksnatur des größeren Teiles der Fondsgelder sich ergeben würden, zu dienen.

An sich ist der Fonds in quali et quanto beschränkt durch Art. 1: in ersterer Richtung dadurch, daß demselben nur die Erträge außerordentlicher Nutzungen in dem in der Begründung zum Gesetzesartikel gegebenen Sinn und bis zum Betrag von $300000 \mathrm{fm}$, sowie die Erträgnisse größerer Holzanfälle infolge von Naturereignissen, soweit sie nicht durch eine Ermäßigung der ordentlichen Nutzungen ausgeglichen werden können, zufließen. Zeitliche Beschränkung ist durch Art. 4 gegeben - bis 31. März 1917.

Der Fonds wurde schon in den ersten 4 Jahren seines Bestehens durch Nutzung der $300000 \mathrm{fm}$ in außerordentlicher Weise gefüllt und geben hierüber die Erläuterungen zum Hauptfinanzetat für 1909/10 (Kapitel 112 Titel 1) Aufschluß.

Daselbst ist aufgeführt:

Der Stand des Forstreservefonds wird nach Abschluß des Wirtschaftsjahrs 1908 folgender sein:

\begin{tabular}{|c|c|c|c|c|c|}
\hline & & $\mathrm{fm}$ & $\begin{array}{l}\text { Roheinnahmen } \\
16\end{array}$ & $\begin{array}{c}\text { Ausgaben } \\
\sqrt{16}\end{array}$ & $\operatorname{Reineinnahmen~}_{\mathbb{N} 6}$ \\
\hline 1905 & . & 15000 & 251700 & 48000 & 203700 \\
\hline 1906 & . & . 120000 & 2028000 & 384000 & 1644000 \\
\hline 1907 & . & . 150000 & 2665500 & 480000 & 2185500 \\
\hline 1908 & . & 15000 & 255000 & 50250 & 204750 \\
\hline zl & & 300000 & 5200200 & 962250 & 4237950 \\
\hline
\end{tabular}


Eine Inanspruchnahme des Fonds ist dank der Gunst der Zeitsn bis jetzt nicht erforderlich geworden, droht auch für die laufendon Etatsjahre 1909 und 10 nicht, so daß wir voraussichtlich über dis Hälfte der Geltungsdauer des Gesetzes ohne Eingriff in den Fondsbestand auskommen werden. Wie kein wesentlicher Preisrückgang eingetreten ist, so sind wir auch von Naturereignissen mit erheblicheren Holzanfällen verschont geblieben. Erhalten sich die Verhältnisse fortdauernd günstig, so haben wir Aussicht, auf 31. März 1917 vor gefülltem oder doch noch beträchtlichem Fonds zu stehen und über seine Verwendung verfügen zu können. Um diese Verwendung in die richtigen Bahnen zu lenken und im Zusammenhang damit die Reservebildung auf die ihr gebührende breite und richtige Grundlage zu stellen, wäre zu untersuchen, in welcher Weise die Umgestaltung des Reservefondsgesetzes zu bewirken wäre.

\section{Der weitere Ausbau der Fondsgesetzgebung.}

Um den in Ziffer 1 aufgestellten Forderungen gerecht zu werden, empfiehlt es sich nicht, die in den Motiven zu Artikel 4 in Aussicht genommene Verlängerung des bestehenden Fondsgesetzes einzuleiter. Dieses Gesetz erfordert nach Inhalt, Zweck und Wirkungsdaner solche Umgestaltungen, daß Neufassung entschieden rorzuziehen ist.

Als Grundlagen für das neue Gesetz kommen in Betracht:

a) Beschränkung der Gültigkeitsdauer des Gesetzes ron 1905 auf einen bestimmten Zeitpunkt z. B. auf 31. März 1911 oder 1912 und Bestimmung, daß das bis dahin vorhandene Vermögen dem nach den Bestimmungen des neuøn Gesetzes zu begründenden Fonds zugeht.

b) Der neue Fonds wird in der Art begründet, daß die in den Wirtschaftsplänen als außerordentliche Nutzungen bezeichneten Fällungen bezw. deren Erträge, sowie die Mehrerträge der laufenden Verwaltung gegenüber den Etatssätzen, event. abzüglich der Beträge, welche zur Deckung von Mehrausgaben innerhalb des Forstetats erforderlich werden, dem Fonds zugehen.

c) In dem Fonds werden zunächst zwei Abteilungen gebildet:

\section{A. Grundstocksabteilung,}

welcher neben dem Vermögen des bestehenden Reservefonds alle aus außerordentlichen Nutzungen der Wirtschaitspläne fließenden Reinerträge zugehen, ferner die Scheidholzanfälle bezw. deren Rein- 
erträge in Beständen späterer Perioden, insbesondere beim Eintreten schädlicher Naturereignisse.

\section{B. Betriebsabteilung,}

welcher die Überschüsse der laufenden Verwaltung zufließen.

d) Als Zweck der Fondsabteilungen ist zu bestimmen:

Für Abteilung A: die Vergrößerung, Verbesserung und Sicherung des Naturalgrundstocks einerseits, die Deckung von Ertragsausfällen der nächsten bezw. einer späteren Periode andererseits. Eine Scheidung der beiden Unterabteilungen dieses Fonds ist angezeigt, insbesondere um die Erfüllung des letztgenannten Zweckes sicher zi stellen und auch deshalb, weil hier Kapital und Zinsen unverändert anzuwachsen haben.

Für A bteilung B: die Ausgleichung von Schwankungen der Preise und der Nutzungsgrößen zur Sicherung des Forstetats bezw. der staatlichen Etatswirtschaft und im Interesse kaufmännischen Betriebes der Forstverwaltung.

e) Der neue Forstreservefonds ist als dauernde und selbständige Einrichtung des Staatshaushalts ähnlich derjenigen des allgemeinen Grundstocks der staatlichen Vermögensverwaltung zu schaffen. Für denselben ist ein Betriebsplan von 2 zu 2 Jahren zu fertigen und jeweils dem Hauptfinanzetat anzuschließen bezw. der ständischen Beratung zu unterstellen. Dieser Betriebsplan soll es jedoch nicht ausschließen, im Fall außerordentlichen Geldbedarfs für die Zwecke der Fondsabteilung A, z. B. zu größeren Waldkäufen, Ablösungen von Holzgerechtigkeiten usw., die entsprechenden Entnahmen aus dem Fonds, event. vorbehältlich der ständischen Verabschiedung einzuleiten.

\section{Die Fiillung des Fonds und das Tempo hierfiir.}

Behufs Ausführung des Gesetzes ist eine wichtige Frage. in welcher Weise und insbesondere in welchem Tempo statten wir den Fonds aus? Wir werden hierbei die beiden Hauptabteilungen des letzteren wieder abgesondert betrachten müssen.

\section{A. Die Grundstocksabteilung.}

Die erste Einlage in dieselbe hätte das zu Beginn der Wirksamkeit des neuen Gesetzes vorhandene Vermögen des seitherigen Reservefonds zu bilden.

Es könnte natürlich auch in Frage kommen, in Hinblick auf 
den Hauptzweck des alten Fonds das Vermögen zwischen Abtrilung $A$ und $B$ zu teilen, insbesondere um der Abteilung $B$ sofort ein gewisses Betriebskapital zu geben.

Den weiteren Zufluß zu A hätte die Erneuerung der sämtlichen Wirtschaftspläne der Staatswaldungen (derzeit 143) zu regeln. Schon aus Kapitel I und II geht herror, daß nach meinem Dafürhalten eine genügend genaue Fondsbildung n ur durch Revision der Wirtschaftspläne auf den vorgeschlagenen Grundlagen und mit Hilfe des zu gründenden Einrichtungsbureaus ermöglicht wird. Diese Revisionen sollen aber keine außerordentlichen sein, sondern zu ihrer regelmäßigen Verfallzeit vorgenommen werden, so daß im ganzen 10 Jahre für Schaffung der Fondsgrundlagen und der Fondsmittel erforderlich würden, ein Zeitraum, welcher in Anbetracht der Bedeutung der Sache, der hohen Werte, um welche es sich handelt, nicht für zu lang zu erachten ist, aber auch keinen wesentlichen lucrum cessans in sich schließt, da ja schon in den bestehenden Wirtschaftsplänen der rechtzeitigen Abnutzung ron Altbeständen meist Rechnung getragen ist, allerdings nicht der Abscheidung ron einem Teil der Erträge zur Fondsbildung. Sodann ist zu bemerken, daß eine beschleunigte Rerision der Wirtschaftspläne auf Kosten der Gründlichkeit ginge und mit dem für das Einrichtungsbureau rorgesehenen Personal nicht ausgeführt werden könnte. Es hätte sofort die Einstellung außerordentlichen Personals zu erfolgen, was erheblich rermehrte Kosten verursacben würde. Der Zeitraum ron 10 Jahren wirkt auch in der Hinsicht günstig, daß der Holzanfall der außerordentlichen Nutzung auf längere Zeit verteilt wird, jedoch nicht ausschließt, wenn die Wirtschattspläne einzelner Bezirke fertig sind, eine Hochkonjunktur des Marktes zu einem Mehrhieb auszunützen.

Von einer raschen Fondsbildung, etwa unter Benutzung der in der Anlage zum Hauptfinanzetat pro 1909 und 10 (Kap. 112 Tit. 1) gegebenen Zahlen und auf Grund der Einrichtungsstatistik, welche 1908 für die Zwecke jener Anlage aufgestellt worden ist, müchte ich abraten. Dieser Statistik fehlt es meines Erachtens an der Sicherheit der Unterlagen. Einmal sind die Flächenunterlagen für Ausscheidung der Altersklassen nicht überall in gleicher Weise erhoben und hinreichend zuverlässig, sodann fehlt es an Regelung der Abnutzungsflächen nach einheitlichen Grundsätzen. Weiterhin ist die Berechnung der Normalnutzung für das ganze Land mit Hilfe des sogenannten Normalzuwachses für die einzelnen Holzarten auf 
den verschiedenen Standorten und mit Hilfe der Reduktion dieses Zuwachses auf den wirklichen Zuwachs wegen der hierin enthaltenen Schätzung des Reduktionsfaktors sehr anfechtbar. Einen genügenden Maßstab für Feststellung des Schlußgrades haben wir nur für dia stammweise aufgenommenen haubaren Bestände, nicht aber für das Gros der übrigen Bestände. Die Lokalertragstafeln sind noch zu wenig entwickelt, als daß eine Reduktionsziffer mit befriedigender Genauigkeit für alle Bestände festgestellt werden könnte.

Ich sehe jene Ertragszahlen, welche aus der Statistik abgeleitet sind, als für gewisse Zwecke brauchbare Näherungswerte an, aber nicht als genügende Grundlage für Ausscheidung zwischen Nutzung der laufenden Verwaltung und der Reserveverwalturg:

Auch die Zahlen von Forstrat Müller-Stuttgart in dem Aufsatz: „Über die Holznutzung und den Altersklassenstand in den württembergischen Staatsforsten" (Allg. Forst- und Jagdztg. 1909 S. 265) können meines Erachtens nur Näherungswert beanspruchen, wenn sie auch wertvolle Fingerzeige für Reservebildung bieten, denen der Genannte selber in dem Aufsatz: „Über Holzvorratsüberschüsse und Anlage von Reservefonds für Staatsforsten" (das. S. 297) weiter nachgegangen ist.

Sowohl die Zahlen der angeführten Etatsanlage, wie diejenigen von Müller wollen die vorliegende Frage auf summarischem Weg und in kürzester Zeit lösen, aber jene Zahlen haben nicht zu unterschätzende Schattenseiten, selbst wenn die Berechnungen nach Waldgebieten getrennt aufgestellt werden.

Ich fürchte, die waldbaulichen Gesichtspunkte werden bei Festsetzung der außerordentlichen Nutzungen ohne Zusammenhang mit dem einzelnen Wirtschaftsplan mehr oder weniger hintangesetzt. Wir vermehren die Kahlflächen und an Orten, auf welchen waldbaulich und schließlich auch finanziell langsameres Vorgehen angezeigt wäre!

Des weiteren fürchte ich, daß diє außerordentlichen Nutzungen nicht immer in den Beständen des größten Überalters und da, wo der Zuwachs am meisten zurückgegangen ist, erhoben werden und zwar mangels genauerer Untersuchung im Bestand. Solche Untersuchungen werden bei Erneuerung der Wirtschaftspläne, nötigenfalls mit Hilfe des Zuwachsbohrers, Platz greifen müssen.

Endlich spielt auch die Waldarbeiterfrage herein, welche die Verteilung der Starkholznutzungen auf einen gewissen Zeitraum angezeigt erscheinen lassen kann. 
Von all diesen (resichtspunkten aus möchte ich für die weitere Reservebildung zunächst einen Zeitraum ron 10 Jahren fordern. damit alle einschlägigen, zum Teil sehr verwickelten Fragen in Ruhe, systematisch und im Zusammenhang mit dem Gefüge des einzelnen Wirtschaftsplans gelöst werden können. Auf diese Weise würde jährlich für 14-15 Forstbezirke über die Möglichkeit von Zuweisungen zum Reservefonds entschieden, so daß die weitere Fondsbildung genügend rasch ins Leben treten würde - vorausgesetzt, daß das Einrichtungsbureau gleichzeitig mit dem neuen Fondsgesetz geschaffen wird.

Ich sagte oben: „zunächst" einen Zeitraum von 10 Jahren und habe hierbei unterstellt, daß die Reservebildung künftig bei jeder Hauptrevision des Wirtschaftsplans ebenfalls revidiert wird, wie ja auch die Nutzungsfläche neu zu regeln ist und erent. neue Bestände mit Überalter zur Nutzung eingestellt werden. Dieser Punkt weist auch darauf hin, den Reservefonds als eine dauernde Einrichtung zu begründen.

In Vorstehendem habe ich mir die Aufgabe gestellt, ein Vorgehen in der Reservebildung zu entwickeln, welches rom theoretischen Standpunkt aus richtig erscheint, durch die Ausführungen in Kapitel I und II begründet ist und in seinen praktischen Wirkungen, insbesondere in waldbaulicher Hinsicht, tunlich vor Nachteilen bewahrt.

Sollten jedoch fin anzpolitische Erwägungen rorliegen, welche eine raschere Füllung des Fonds bedingen und größere Erhebungen für die nächsten Jahre oder das nächste Jahrzehnt rorsehen, so läßt sich ein annäherndes $\mathrm{Ma}$ ßür diese außerordentlichen Nutzungen folgendermaßen gewinnen, indem ich die Formeln der Kapitel I und Il für Berechnung der Abnutzungsflächen des nächsten Jahrzwanzig auf die Staatsforste der einzelnen Waldgebiete des Landes, wie der Gesamtfläche anwende, weiterhin als ertragsfähige Flächen des Holzgrundes und für deren prozentuale Verteilung auf die Altersklassen die in der Anlage zu Kapitel 112 des württembergischen Hauptfinanzetats für 1909 und 10 enthaltenen Zahlen unterstelle.

Die ertragsfähigen Waldflächen betragen und rerteilen sich nach dem Alter, wie folgt: 


\begin{tabular}{|c|c|c|c|c|c|c|c|c|c|c|c|}
\hline \multirow{3}{*}{\multicolumn{4}{|c|}{$\begin{array}{r}\text { Eitragsfähige Fläche } \\
\text { grundes }\end{array}$}} & \multirow{3}{*}{ des } & \multirow{3}{*}{$\begin{array}{l}\text { Holz- } \\
\text { ha }\end{array}$} & \multicolumn{6}{|c|}{$\begin{array}{l}\text { Yon der ertragsfähigen Fläche entfallen auf } \\
\text { die Altersklassen }\end{array}$} \\
\hline & & & & & & $\begin{array}{c}\mathbf{f} \\
\text { u. } m e h r\end{array}$ & e & $d$ & $\mathrm{c}$ & $\mathrm{b}$ & a \\
\hline & & & & & & \multicolumn{6}{|c|}{ Prozente } \\
\hline Schwarzwald & . & & & - & 44338 & 23 & 16 & 12 & 11 & 16 & 22 \\
\hline Unterland . & . & & . & . & 41070 & 8 & 11 & 17 & 18 & 22 & 24 \\
\hline Nordostland . & . & & . & . & 35477 & 10 & 10 & 14 & 20 & 26 & 20 \\
\hline Alb. . . & . & & 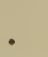 & . & 43701 & 8 & 14 & 17 & 19 & 21 & 21 \\
\hline Oberschwaben & . & - & - & . & 22713 & 10 & 12 & 13 & 16 & 26 & 23 \\
\hline Sa. Staatsforste & & • & 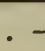 & $\therefore$ & 187299 & 12 & 13 & 15 & 16 & 22 & 22 \\
\hline
\end{tabular}

Die Nutzungsflächen fürs nächste Jahrzwanzig und die aus denselben sich mutmaßlich ergebenden Derbholzerträge sind in nachstehender Tabelle berechnet:

(Siehe Tabelle S. 45.)

Aus der Tabelle geht hervor, daß für die nächsten 20 Jahre eine jährliche Hauptnutzung von

$$
\text { rund } \div 800000 \mathrm{fm} \text {, }
$$

woron jedoch $\div 92 \%=736000 \mathrm{fm}$ auf ordentliche Nutzung, $8 \%=64000 \mathrm{fm}$ auf außerurdentliche Nutzung entfallen, für zulässig zu erachten ist. - Vergleichen wir hiermit die in der Etatsanlage pro 1909 und 1910 berechnete Hauptnutzung von

$$
\therefore 857460 \mathrm{fm} \text {, }
$$

so erhellt, daß in dieser Nutzung schon eine immerhin erhebliche außerordentliche Nutzung bezw. Vorratsabtragung steckt, welche nach Analogie der obigen Berechnung $\mathrm{zu} 8 \%$ $=68600 \mathrm{fm}$ geschätzt werden kann, so daß auf die ordentliche Nutzung $\div 788860 \mathrm{fm}$ entfielen.

Wollen wir die derzeitige wirtschaftsplanmäßige Hauptnutzung von $\div 857460 \mathrm{fm}$ im nächsten Jahrzehnt forterheben, so ergibt sich nach dessen Ablauf in der Gesamtnutzung:

$$
\begin{gathered}
\text { an ordentlicher } \\
\text { Nutzung }
\end{gathered}
$$

an außerordentlicher

Nutzung

ein Hat . . . . . . . ca. $7888600 \mathrm{fm} \quad \therefore 686000 \mathrm{fm}$ bei einem Soll von . . - ca. 7360000 " $\quad \div 640000$ " als Mehr gegenüber dem Soll $\div$ ca. $528600 \mathrm{fm} \quad \therefore \quad 46000 \mathrm{fm}$ 


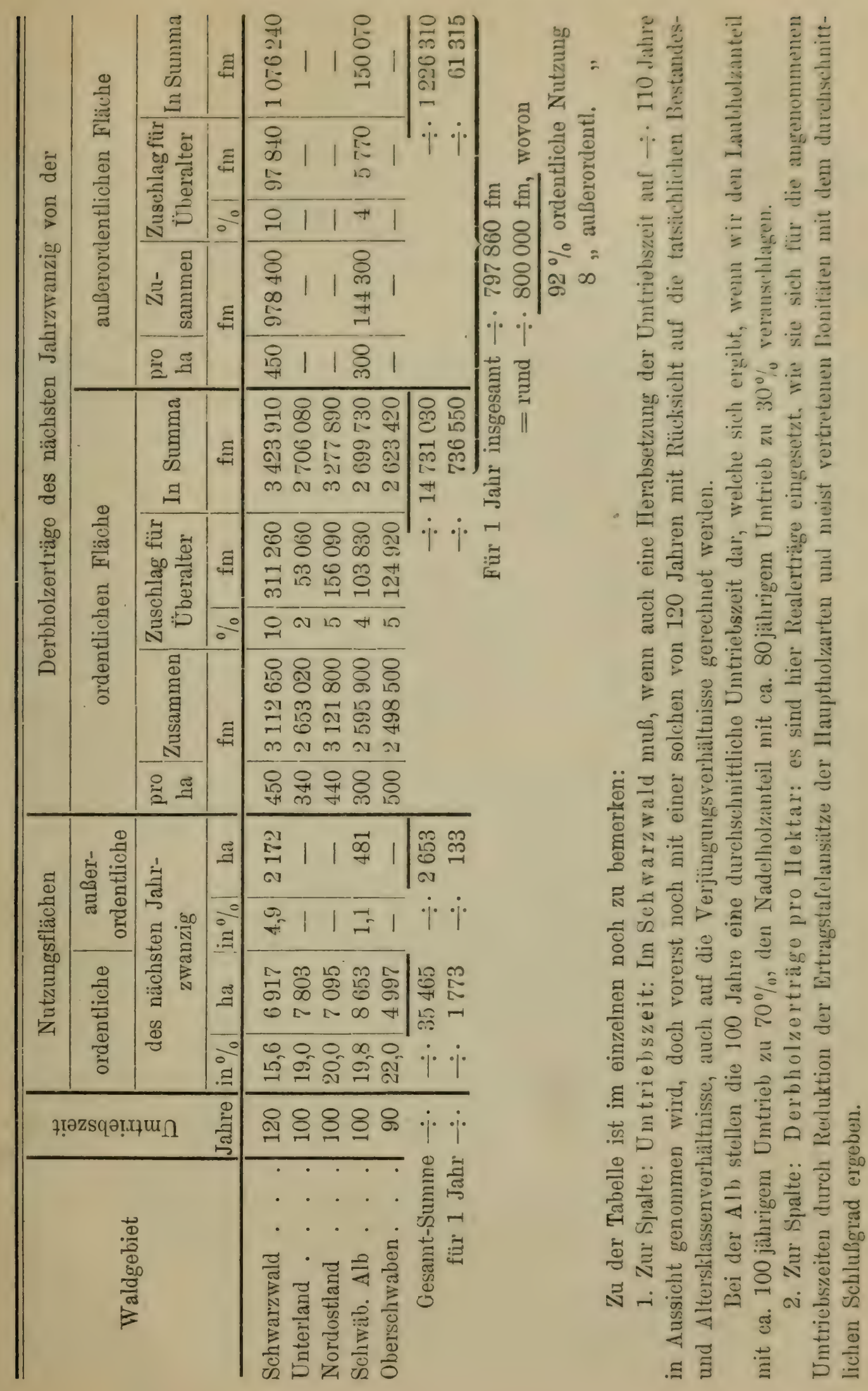


Es bleiben fürs 2. Jahrzehnt noch übrig

an ordentlicher

Nutzung

Hauptnutzung . . . . . $\div$ ca. $6831400 \mathrm{fm}$

für ein Jahr .
- $\quad \therefore$ ca. 683140

zusammen $\div 742540 \mathrm{fm}$ Jahresnutzung, an außerordentlicher

Nutzung $\therefore 594000 \mathrm{fm}$ $\therefore \quad 59400$

was einem Rückgang der letzteren im 2. Jahrzehnt um rund $\therefore 115000 \mathrm{fm}$ gleichkäme.

Es kann nun bei Forterhebung der derzeitigen wirtschaftsplanmäßigen Hauptnutzung von $\div 857460 \mathrm{fm}$ im nächsten Jahrzehnt sich fragen, ob nicht die fürs 2. Jahrzehnt vorstehend berechnete außerordentliche Nutzung von $\div 594000 \mathrm{fm}$ ins 1. Jahrzehnt als außerordentliche Nutzung zum Reservefonds vorgeschoben werden könnte? Diese Erage ist vom finanziellen Standpunkt aus zu bejahen, vom waldbaulichen Standpunkt aus als zweifelhaft zu bezeichnen, wie oben schon hervorgehoben worden ist. Die Zweifel bewegen sich in der Richtung, daß im Fall jener Vorschiebung sich unerwünschte waldbauliche Folgen, insbesondere die Vermehrung größerer Kahlhiebe, das weitere Umsichgreifen der reinen Fichtenbestände mit ihren Gefahren, zumal im Gebirge, zeigen.

Läßt man diese Zweifel und Bedenken hinter dem rein finanziellen Gesichtspunkt zurücktreten, so kann eine Nutzung von in $\max$ :

$\therefore 600000 \mathrm{fm} \mathrm{zum} \mathrm{Reservefonds}$

im Laufe des nächsten Jahrzehnts zugegeben werden neben Forterhebung der derzeitigen Hauptnutzung. Diese Reservenutzung schließt aber einen wesentlichen Rückgang der planmäßigen Hauptnutzung im 2. Jahrzehnt, nämlich auf $\div$ rund $683000 \mathrm{fm}=\mathrm{um}$ rund $174460 \mathrm{fm}$ in sich. Diese Mindernutzung würde, wenn wir als erntekostenfreien Durchschnittserlös für $1 \mathrm{fm}$ von $\div 15 \mathrm{M}$ annehmen, einen Ausfall am Geldertrag ron $\div$ 2,6 Mill. Mark pro Jahr darstellen.

Eine Ausgleichung könnte allerdings dadurch geschaffen werden, daß seinerzeit entsprechende Vorschiebungen vom 3. ins 2. Jahrzehnt stattfänden. Die Möglichkeit hierfür ist gegcben, da für zwei Waldgebiete, den Schwarzwald und die Alb, bei Berechnung der außerordentlichen Nutzungsflächen fürs nächste Jahrzwanzig in der Tabelle oben ein Teil der Altholzflächen (e $+\mathrm{f}$-Bestände): im ersteren Fall 
$2,5 \%$, im letzteren $1,1 \%$, je der ertragsfähigen Flächen, zur $\mathrm{Ab}$ nutzung im übernächsten Jahrzwanzig bezw. im 3. Jahrzehnt vorbehalten worden sind. Es geschah dies nicht nur aus waldbanlichin Gründen, sondern auch mit Rüicksicht darauf, dafj die Vertretung der 60-80jährigen (d-)Bestände in den Staatsforsten des Landes, besonders im Schwarzwald, eine mangelhafte, unternormale ist. Werden die betreffenden zurückgestellten Flächen als auferordentliche Nutzungsfläche fürs nächste Jahrzwanzig eingestellt, so wäre die Nutzung zum Reservefonds mit $600000 \mathrm{fm}$ mohr als ausgeglichen.

Aber eines möchte ich auf Grund der Tabelle \$. t5 noch ausdrucklich hervorheben, daß nach meiner formelmäßigen Berechnung der Abnutzungsflächen, also abgesehen vom Überalter der Bestände. außerordentliche Nutzungen zum Reservefonds nur im Schwarzwald und zu einem kleinen Teil auch auf der Alb begründet sein dürften.

\section{B. Die Betriebsabteilung.}

Schon oben wurde die Möglichkeit erörtert, dieser Abteilung durch Zuscheidung eines Teils vom Vermögen des bestehenden Fonds ein Betriebskapital zu geben, wenn es auch grundsätzlich für richtiger zu erachten ist, den vorwiegend aus außerordentlichen Nutzungen begründeten alten Fonds ganz der Grundstocksabteilung zuzuführen. Es könnte das Vermögen vielleicht in der Weise geteilt werden, daß der auf Vorratsabnutzung sich gründende Teil der Grundstocksabteilung des neuen Fonds, der vom Mehranfall an Durchforstungsmaterial herrührende Teil der Betriebsabteilung zugeschieden würde.

Im übrigen sind die Zeitumstände günstig, den Fonds der Betriebsabteilung aus Überschüssen der laufenden Verwaltung zu begründen. Vom Etatsjahr 1909 werden sich wahrscheinlich Überschüsse ergeben und vom Jahr 1910 sind solche bei den derzeitigen Nutzholzerlösen in erhöhtem Maß zu erwarten. Der Preisstand würde es sogar rechtfertigen, Vorauserhebungen für die laufende Verwaltung, also an ordentlicher planmäßiger Nutzung, und insbesondere in denjenigen Landesteilen einzuleiten, in welchen außerordentliche Nutzungen voraussichtlich nicht stattfinden werden. Derartige Vorauserhebungen schlössen auch Zinsengewinn in sich.

In die Überschüsse dürften auch die MIehrertrïge ron Durchforstungen einzubeziehen sein, dies um so mehr, als sich bei den 
nach 5-10 Jahren auf denselben Flächen wiederkehrenden Durchforstungen Mindererträge gegenüber den Voranschlägen ergeben können.

Man kann ferner daran denken, diesem Fondsteil ron den Scheidholzanfällen späterer Perioden diejenigen Quoten zuzuweisen, welche sich im Rahmen des Durchschnittsanfalls halten bezw. voraussichtlich einen Ausfall am Haubarkeitsertrag nicht geben werden. Doch spielt diese Frage in eine andere herein, in diejenige der Verrechnung solcher Holzanfälle unter Haupt- oder Zwischennutzung, worauf hier nicht eingegangen werden will. In diesem Kapitel war es mir darum zu tun, die Reservebildung nach allen Richtungen zu erwägen und weiteren Anlaß zur Erörterung der Fragen zu geben.

Die Erneuerung des Reservefondsgesetzes für die württembergischen Staatsforste auf richtiger und genügend breiter Grundlage halte ich für eine der wichtigsten, wenn auch schwierigsten forstpolitischen Aufgaben der nächsten $\mathrm{Zu}$ kunft und die Lösung derselben steht im engsten Zusammenhang mit der Schaffung einer geeigneten Organisation der Forsteinrichtung. $\mathrm{Zu}$ jener Lösung haben die forstlichen und finanziellen Sachverständigen harmonisch zusammenzuwirken, damit in dem neuen Reservefonds eine Art "Staatliche Forstbank" erwachse, deren „fundus instructus" in erster Linie zur Hebung des heimischen Forstwesens und von dessen Holz- bezw. Gelderträgen diene, in zweiter Linie zur Deckung etwaiger späterer Ertragsausfälle, weiterhin in gewissem Rahmen zur Ermöglichung des kaufmännischen Betriebes der Forstverwaltung und zur Sicherung der staatlichen Etatswirtschaft.

Noch ein weiterer Ausblick! Auch die Körperschaften mit größerem Waldbesitz, auf welchen ja die staatlichen Forsteinrichtungsvorschriften gleichfalls Anwendung finden, werden sich wohl durch den Vorgang bei den Staatsforsten veraulaßt sehen, der rechtzeitigen Abnutzung von Vorratsüberschüssen in Verbindung mit Fondsbildung näher zu treten, in welcher Richtung in einigen Fällen erfreuliche Anfänge gemacht sind.

Möge nun auch für die Körperschaftsforste in dem neuen Fondsgesetz für die Staatsforste ein glücklicher Vorgang geschaffen werden! 


LIBRARY

FACULTY OF FORESTRY

UNIVERSITY OF TORONTO

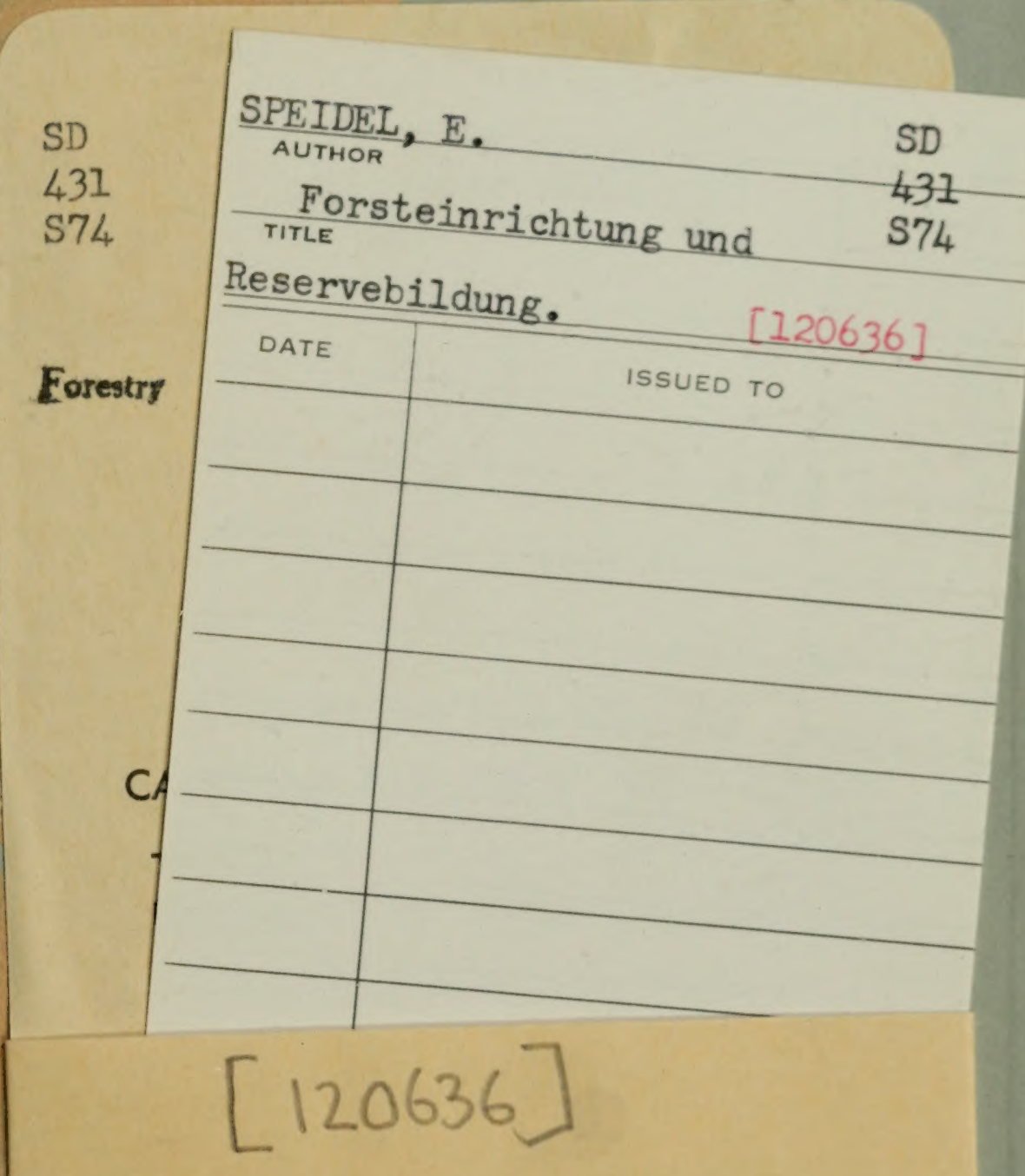




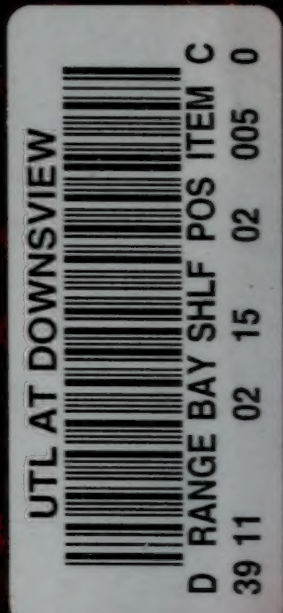

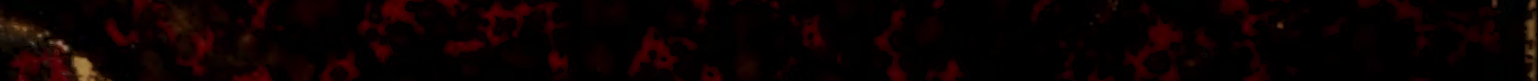

